\title{
SEPARATRIX SPLITTING IN 3D VOLUME-PRESERVING MAPS*
}

\author{
HÉCTOR E. LOMELíi AND RAFAEL RAMíREZ-ROS ${ }^{\ddagger}$
}

\begin{abstract}
We construct a family of integrable volume-preserving maps in $\mathbb{R}^{3}$ with a bidimensional heteroclinic connection of spherical shape between two fixed points of saddle-focus type. In other contexts, such structures are called Hill's spherical vortices or spheromaks. We study the splitting of the separatrix under volume-preserving perturbations using a discrete version of the Melnikov method.

Firstly, we establish several properties under general perturbations. For instance, we bound the topological complexity of the primary heteroclinic set in terms of the degree of some polynomial perturbations. We also give a sufficient condition for the splitting of the separatrix under some entire perturbations. A broad range of polynomial perturbations verify this sufficient condition. Finally, we describe the shape and bifurcations of the primary heteroclinic set for a specific perturbation.
\end{abstract}

Key words. Separatrix splitting, volume-preserving maps, primary heteroclinic set, Melnikov method, bifurcations

AMS subject classifications. 34C37, 34C23, 37C29, 33E20

1. Introduction. A fundamental question in dynamical systems is the effect that small perturbations of a dynamical system cause on its unperturbed invariant sets. The most studied unperturbed invariant sets are tori and stable/unstable invariant manifolds of hyperbolic sets. Usually, the unperturbed dynamical system is integrable and has separatrices; that is, its stable and unstable invariant manifolds overlap. After a generic perturbation, the perturbed stable and unstable invariant manifolds intersect transversely, which give rise to the onset of chaos, through the creation of Smale horseshoes. This phenomenon is known as the problem of splitting of separatrices. A widely used technique for detecting such intersections is the Melnikov method.

Our goal is to apply the Melnikov method to the splitting of separatrices in the discrete volume-preserving framework. Similar questions have been considered before. However, we believe this is the first time that detailed analytical results about the structure of the primary heteroclinic set and its bifurcations are established for specific maps. This represents a step forward with respect to previous works [22, 23], in which once written down a formula for the Melnikov function in terms of an infinite series, the approach becomes mainly numerical, because of the technical difficulties that obstruct the analytical one. Here, we have overcome some of these difficulties using basic tools: complex variable theory, quasielliptic functions, homology, and several algebraic tricks. Nevertheless, we have not been able to find an explicit expression of the Melnikov function in terms of elementary functions for any specific perturbation. In opposition, such explicit expressions (in terms of elliptic functions) are known for almost twenty years in the discrete area-preserving setting $[17,13]$.

This study is interesting because volume-preserving maps are the simplest, and most natural higher-dimensional versions of the much-studied class of area-preserving maps. The infinite dimensional group of volume-preserving diffeomorphisms on $\mathbb{R}^{3}$

\footnotetext{
*January 11, 2008

${ }^{\dagger}$ Department of Mathematics, Instituto Tecnológico Autónomo de México, Mexico, DF 01000 (lomeli@itam.mx). HL was supported in part by Asociación Mexicana de Cultura.

${ }^{\ddagger}$ Departament de Matemàtica Aplicada I, Universitat Politècnica de Catalunya, Diagonal 647, 08028 Barcelona, Spain (Rafael.Ramirez@upc.edu). RR-R was supported in part by MCyT-FEDER grant MTM2006-00478.
} 
is at the core of the ambitious program to reformulate hydrodynamics [3]. Volumepreserving maps arise in a number of applications such as the study of the motion of Lagrangian tracers in incompressible fluids or of the structure of magnetic field lines [18, 19, 32, 29]. Experimental methods have only recently been developed that allow the visualization of particle trajectories in spatial fluids [27, 31].

Given a system with a heteroclinic connection between two hyperbolic fixed points, the Melnikov function computes the rate at which the distance between the manifolds changes with a perturbation. After the introduction of the Melnikov method for periodic perturbations of one-degree-of-freedom Hamiltonian systems, many different versions appeared, most of them in continuous settings (flows). For instance, there are versions for three-dimensional incompressible flows in $[28,5,6]$.

There exist also discrete versions, in which the Melnikov function is no longer an integral, but an infinite sum whose domain is the unperturbed connection. The first steps towards a discrete Melnikov theory were performed for area-preserving maps [16, 17, 13, 20], and next, for symplectic maps [14], for twist maps [21], for general $n$-dimension diffeomorphisms $[8,24]$, and for spatial billiard maps [12]. Finally, volume-preserving maps have been considered in $[22,23]$. These papers deal with codimension-one heteroclinic connections between fixed points of saddle-focus type and between hyperbolic invariant circles, respectively. The current paper is a natural continuation and uses some of their ideas.

We shall construct a family of integrable volume-preserving maps $f: \mathbb{R}^{3} \rightarrow \mathbb{R}^{3}$ with a bi-dimensional heteroclinic connection between two fixed points. This family is derived from another family of integrable planar maps introduced by McMillan [26]. The same construction can be found in [22], but we have decided to study a completely new family to minimize the overlap with previous works. Besides, the new family has a purely rational character, whereas the previous one contains trigonometric terms. This can be important for numerical computations using a multiple-precision arithmetic.

The bi-dimensional separatrix has a spherical shape and the fixed points are its "north pole" $p_{+}$and its "south pole" $p_{-}$. Our integrable maps depend on two parameters: a characteristic exponent $h>0$ and a frequency $\omega \in \mathbb{T}$. These names refer to the fact that

$$
\operatorname{spec}\left[D f\left(p_{ \pm}\right)\right]=\left\{\mathrm{e}^{ \pm 2 h}, \mathrm{e}^{\mp h+\mathrm{i} \omega}, \mathrm{e}^{\mp h-\mathrm{i} \omega}\right\}
$$

Thus, the fixed points are of saddle-focus type, the characteristic exponent measures the hyperbolicity of the map, and the frequency quantifies the rotation speed of the trajectories on the separatrix. There exists also an one-dimensional straight heteroclinic connection between the fixed points. The same configuration appears in fluid dynamics under the name of Hill's spherical vortex or bubble-type vortex breakdown [32], and as a model for the magnetic field of stars, in which case it is called spheromak [25]. From a more theoretical point of view, we note that the integrable normal forms associated to families of volume-preserving flows with a Hopf-zero singularity have the same structure in the phase space [11].

In general, volume-preserving perturbations split the separatrix, but the perturbed stable and unstable manifold still intersect along one-dimensional heteroclinic curves, which can be vertical, equatorial or bubble-type ones. This terminology is borrowed from [22]. Vertical curves are those heteroclinic intersections whose endpoints are both fixed points. Due to the rotational dynamics of the unperturbed map, these curves look like spirals connecting both poles when there is swirl; that is, when $\omega \neq 0$. On the contrary, equatorial and bubble-type curves are closed curves that do 

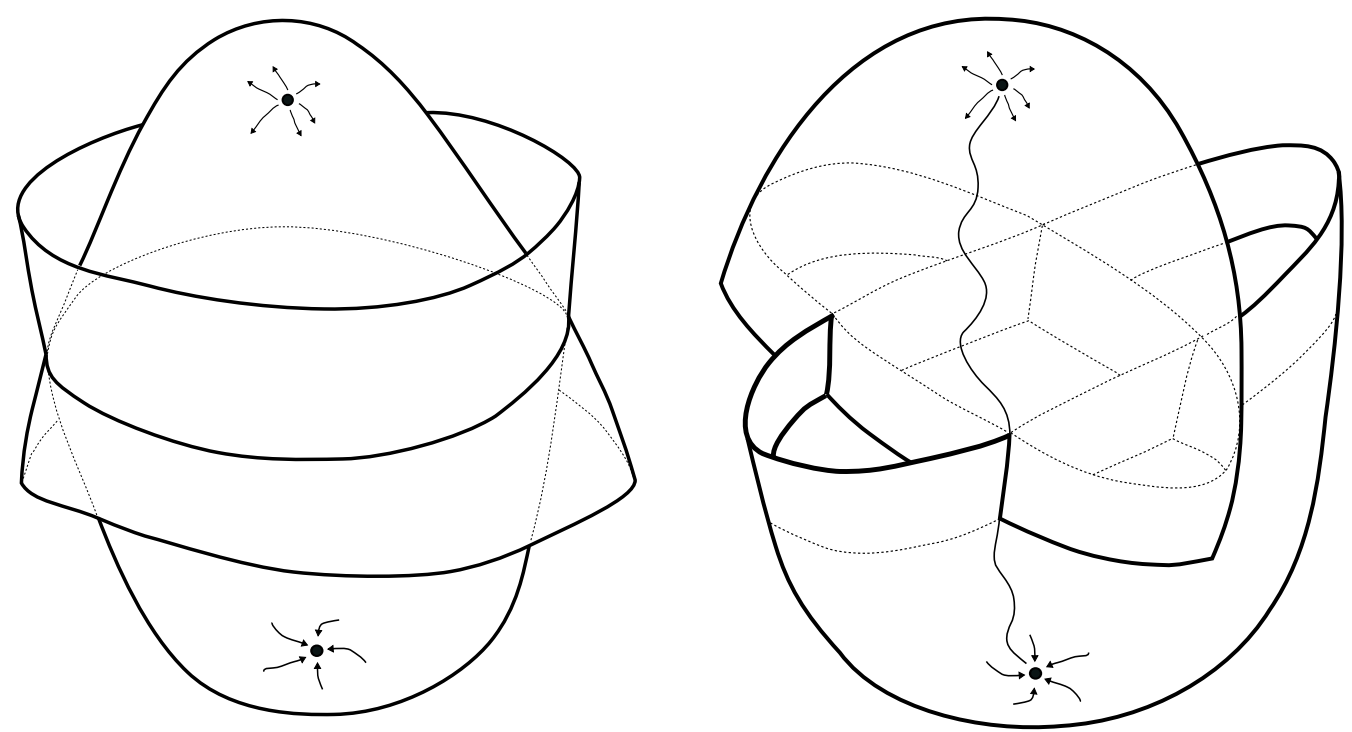

FIG. 1. In this figure we illustrate two possible intersections that appear as heteroclinic intersections of the stable and unstable manifolds that we will be considering. On the left, we have an equatorial intersection. On the right a vertical intersection.

not approach the poles; in particular, they can not appear in autonomous flows. The difference between equatorial curves and bubble-type ones is that the portions of the stable and unstable manifolds delimited by a bubble-type curve encircle a contractible region in $\mathbb{R}^{3}$; that is, a "bubble". See remark 7 and figure 1 for more details.

We shall describe the structure of the set of primary intersections under some perturbations. Roughly speaking, primary intersections are the sets of points where the stable and unstable manifolds "first" meet. In fact, primary intersections are the only intersections that can be followed by the perturbation. In the limit, as $\epsilon \rightarrow 0$, they appear as zeroes of the Melnikov function. Therefore, non-primary intersections are missed by standard Melnikov methods. See [22] for details.

Firstly, we bound the topological complexity of the primary heteroclinic set in terms of the degree of volume-preserving polynomial perturbations of the form

$$
f_{\epsilon}=(\operatorname{Id}+\epsilon \kappa) \circ f, \quad \kappa(x, y, z)=(0, \alpha(x), \beta(x, y)) .
$$

In particular, it turns out that the primary heteroclinic set contains at most $2 n$ vertical curves when $\alpha(x) \in \mathbb{R}_{n-1}[x]$ and $\beta(x, y) \in \mathbb{R}_{n}[x, y]$.

Next, we shall give a sufficient condition for the splitting of the separatrix under some entire perturbations. A broad range of polynomial perturbations verify this condition. For instance, the ones with $\kappa(x, y, z)=(0,0, \beta(x, y))$ for some even polynomial $\beta(x, y)$ of degree $4 l+2$, provided that $\mathrm{e}^{4 k \omega \mathrm{i}} \neq-1$ for $k=1, \ldots, 2 l+1$. In particular, nonresonant frequencies guarantee the breakdown of the unperturbed structure, which is in sharp contrast with some known principles in KAM theory.

Finally, we shall consider the perturbation with $\kappa(x, y, z)=(0, x, 0)$. The primary heteroclinic set under this perturbation consists in four vertical curves for $\omega \neq \pm \pi / 2$, whereas some heteroclinic bifurcations take place at $\omega= \pm \pi / 2$. Unfortunately, we have found a complete proof of these facts only for $h \geq h_{0} \approx 2.28$, but we conjecture, based on numerical experiments, that this picture holds for any $h>0$. The previous upper bound on the number of vertical curves is optimal for this perturbation. 
The proof of each analytical result is based on different tools. The bounds on the topological complexity follow from basic homology theory. The splitting result is obtained through the study of the complex singularities of the Melnikov function, an idea that goes back to Ziglin [34]. The part about bifurcations relies strongly on the fact that the Melnikov function can be expressed in terms of a quasielliptic function of order two. Besides, each part has its own algebraic tricks.

We complete this introduction with a note on the organization of the paper. In $\S 2$, we recall the Melnikov theory for volume-preserving maps. In $\S 3$, we construct the family of integrable volume-preserving maps. Afterwards, we derive an explicit expression for the Melnikov function associated to some volume-preserving perturbations in $\S 4$. The next sections are devoted to bound the topological complexity of the primary heteroclinic set and to establish some sufficient conditions for the splitting. The study about the bifurcations of the primary heteroclinic set under the sample perturbation is contained in $\S 7$. Some analytical details and numerical experiments are relegated to appendix $\mathrm{A}$ and appendix B, respectively.

2. The Melnikov theory for volume-preserving maps. In this section we shall briefly describe the Melnikov theory for volume-preserving maps developed in [22, $23,24]$.

Let $f_{\epsilon}: \mathbb{R}^{3} \rightarrow \mathbb{R}^{3}$ be a family of smooth volume-preserving maps such that the unperturbed map $f=f_{0}$ has two hyperbolic fixed points $a$ and $b$ whose stable and unstable invariant manifolds coincide giving rise to a bi-dimensional saddle connection $\Sigma=W^{\mathrm{u}}(a, f) \backslash\{a\}=W^{\mathrm{s}}(b, f) \backslash\{b\}$, where $W^{\mathrm{u}}(a, f)$ and $W^{\mathrm{s}}(b, f)$ denote the unstable invariant manifold of the point $a$ and the stable invariant manifold of the point $b$, respectively. Both fixed points persist and remain hyperbolic for small $\epsilon$. We want to study how the perturbed invariant manifolds $W^{\mathrm{u}}\left(a_{\epsilon}, f_{\epsilon}\right)$ and $W^{\mathrm{s}}\left(b_{\epsilon}, f_{\epsilon}\right)$ intersect.

Our goal is to describe the topology of the set of primary intersections $P_{\epsilon} \subset$ $W^{\mathrm{u}}\left(a_{\epsilon}, f_{\epsilon}\right) \cap W^{\mathrm{s}}\left(b_{\epsilon}, f_{\epsilon}\right)$. We also pursue to elucidate when the separatrix $\Sigma$ splits under the perturbation; that is, when there is no smooth family of saddle connections $\Sigma_{\epsilon} \subset W^{\mathrm{u}}\left(a_{\epsilon}, f_{\epsilon}\right) \cap W^{\mathrm{s}}\left(b_{\epsilon}, f_{\epsilon}\right)$ such that $\Sigma_{0}=\Sigma$.

We collect in the following theorem the basic Melnikov-like results about this setup. See $[22,24]$

THEOREM 1. Under the previous assumptions, there exists a smooth function $M: \Sigma \rightarrow \mathbb{R}$, called the Melnikov function, with the following properties.

(i) If $\xi_{0}$ is a nondegenerate zero of $M$, then $W^{\mathrm{u}}\left(a_{\epsilon}, f_{\epsilon}\right)$ and $W^{\mathrm{s}}\left(b_{\epsilon}, f_{\epsilon}\right)$ intersect transversely, for $\epsilon$ small enough, at a point $\xi_{\epsilon}=\xi_{0}+\mathrm{O}(\epsilon) \in P_{\epsilon}$.

(ii) If 0 is a regular value of $M$, then the set of primary intersections $P_{\epsilon}$ is, for $\epsilon$ small enough, an one-dimensional submanifold of $\mathbb{R}^{3}$ such that $P_{\epsilon}=M^{-1}(0)+\mathrm{O}(\epsilon)$.

(iii) It is invariant by the unperturbed map: $M \circ f=M$.

(iv) If $f_{\epsilon}$ has a smooth family of:

1. Symmetries $S_{\epsilon}: \mathbb{R}^{3} \rightarrow \mathbb{R}^{3}$ such that $S_{0}(\Sigma)=\Sigma$, then $M \circ S_{0}=M$.

2. Reversors $R_{\epsilon}: \mathbb{R}^{3} \rightarrow \mathbb{R}^{3}$ such that $R_{0}(\Sigma)=\Sigma$, then $M \circ R_{0}=-M$.

3. Saddle connections $\Sigma_{\epsilon} \subset W^{\mathrm{u}}\left(a_{\epsilon}, f_{\epsilon}\right) \cap W^{\mathrm{s}}\left(b_{\epsilon}, f_{\epsilon}\right)$ with $\Sigma_{0}=\Sigma$, then $M \equiv 0$.

The Melnikov function is constructed in such a way that it measures the distance between the perturbed invariant manifolds $W^{\mathrm{u}}\left(a_{\epsilon}, f_{\epsilon}\right)$ and $W^{\mathrm{s}}\left(b_{\epsilon}, f_{\epsilon}\right)$ in first-order. Because of this, the zero-level set $M^{-1}(0) \subset \Sigma$ is strongly related to the primary intersection set $P_{\epsilon}$ and any change in its topology gives rise to some heteroclinic bifurcation, mainly to some tangency between the perturbed invariant manifolds. Besides, a sufficient condition for the splitting of the separatrix is that the Melnikov function is not identically zero. 
We recall some concepts that appear in the theorem above. A point $\xi$ of a manifold $\Sigma$ is a regular point of a smooth function $M: \Sigma \rightarrow \mathbb{R}$ when the differential form $\mathrm{d} M$ does not vanish at $\xi$, whereas $r \in \mathbb{R}$ is a regular value of $M$ if every point in $M^{-1}(r)$ is a regular point. A zero of $M$ is called nondegenerate when it is a regular point. If $r$ is a regular value of $M$, then $M^{-1}(r)$ is an one-dimensional submanifold of $\Sigma$. On the contrary, $M^{-1}(r)$ can be much more complicated if 0 is a singular value, although its subset of regular points is also an one-dimensional submanifold of $\Sigma$. A diffeomorphism $f$ is symmetric when there exists a diffeomorphism $S$ such that $f \circ S=S \circ f$, and then $S$ is called a symmetry of the map $f$. Analogously, $f$ is reversible when there exists a diffeomorphism $R$ such that $f \circ R=R \circ f^{-1}$, and then $R$ is called a reversor of the map $f$ and we denote by $\operatorname{Fix} R=\left\{\xi \in \mathbb{R}^{3}: R(\xi)=\xi\right\}$ the set of its fixed points. These fixed points are called symmetric in the literature.

Symmetries, reversors and symmetric heteroclinic points play an important rôle in the study of (primary) heteroclinic intersections, see [15]. For instance, the set of primary intersections is invariant by symmetries and reversors. Besides, symmetric heteroclinic points persist under reversible perturbations. Concretely, if $f_{\epsilon}$ is $R_{\epsilon}$-reversible and Fix $R_{0}$ is a smooth curve that intersects transversely the saddle connection $\Sigma$ at some point $\xi_{0}$, then there exists a unique point $\xi_{\epsilon}=\xi_{0}+\mathrm{O}(\epsilon) \in P_{\epsilon} \cap$ Fix $R_{\epsilon}$.

Remark 1. If $f_{\epsilon}$ is not $R_{\epsilon}$-reversible, but $f_{\epsilon} \circ R_{\epsilon}-R_{\epsilon} \circ f_{\epsilon}^{-1}=\mathrm{O}\left(\epsilon^{2}\right)$ and $R_{0}(\Sigma)=\Sigma$, then $M \circ R_{0}=-M$. This has to do with the fact that the Melnikov function only measures first-order behaviours. We present an explicit example of this situation in proposition 4 .

In order to apply this theory, we must compute the Melnikov function. This is easier when the unperturbed map has a nondegenerate first integral $I: \mathbb{R}^{3} \rightarrow \mathbb{R}$ and $f_{\epsilon}=($ Id $+\epsilon \kappa) \circ f$ for some map $\kappa: \mathbb{R}^{3} \rightarrow \mathbb{R}^{3}$. Under these assumptions, it is proved in $[22$, Lemma 8] that the Melnikov function is the absolutely convergent series

$$
M=\sum_{k \in \mathbb{Z}}\langle\nabla I, \kappa\rangle \circ f^{k}
$$

This is the formula for Melnikov functions that we shall use in this paper.

We are only interested in perturbations that do not destroy the volume-preserving character of the unperturbed map $f$. This question has a simple answer: $f_{\epsilon}=(\mathrm{Id}+$ $\epsilon \kappa) \circ f$ preserves volume if and only if the differential of the perturbation $\kappa$ is nilpotent everywhere, see [22, Lemma 3]. This allows us to create simple examples of volumepreserving perturbations. For instance, we could take $\kappa(x, y, z)=(0, \alpha(x), \beta(x, y))$ or $\kappa(x, y, z)=(\gamma(y, z), \delta(z), 0)$ for any smooth functions $\alpha, \delta: \mathbb{R} \rightarrow \mathbb{R}$ and $\beta, \gamma: \mathbb{R}^{2} \rightarrow \mathbb{R}$.

3. The maps. In this section we shall construct, following a methodology developed in [22], the perturbed volume-preserving maps $f_{\epsilon}$ that will be studied along the rest of the paper. As a starting point, we shall describe the unperturbed maps $f=f_{0}$ that form a family of integrable volume-preserving maps with a bi-dimensional heteroclinic connection between a couple of hyperbolic fixed points. This integrable family is derived from another family of integrable planar standard-like maps introduced by McMillan [26].

Let $h>0$ be the parameter of the family of planar standard-like maps. Then we consider the quantities $c=\cosh (h / 2)$ and $s=\sinh (h / 2)$, the rational transformation

$$
z \mapsto \phi(z)=\frac{c z+s}{c+s z}
$$




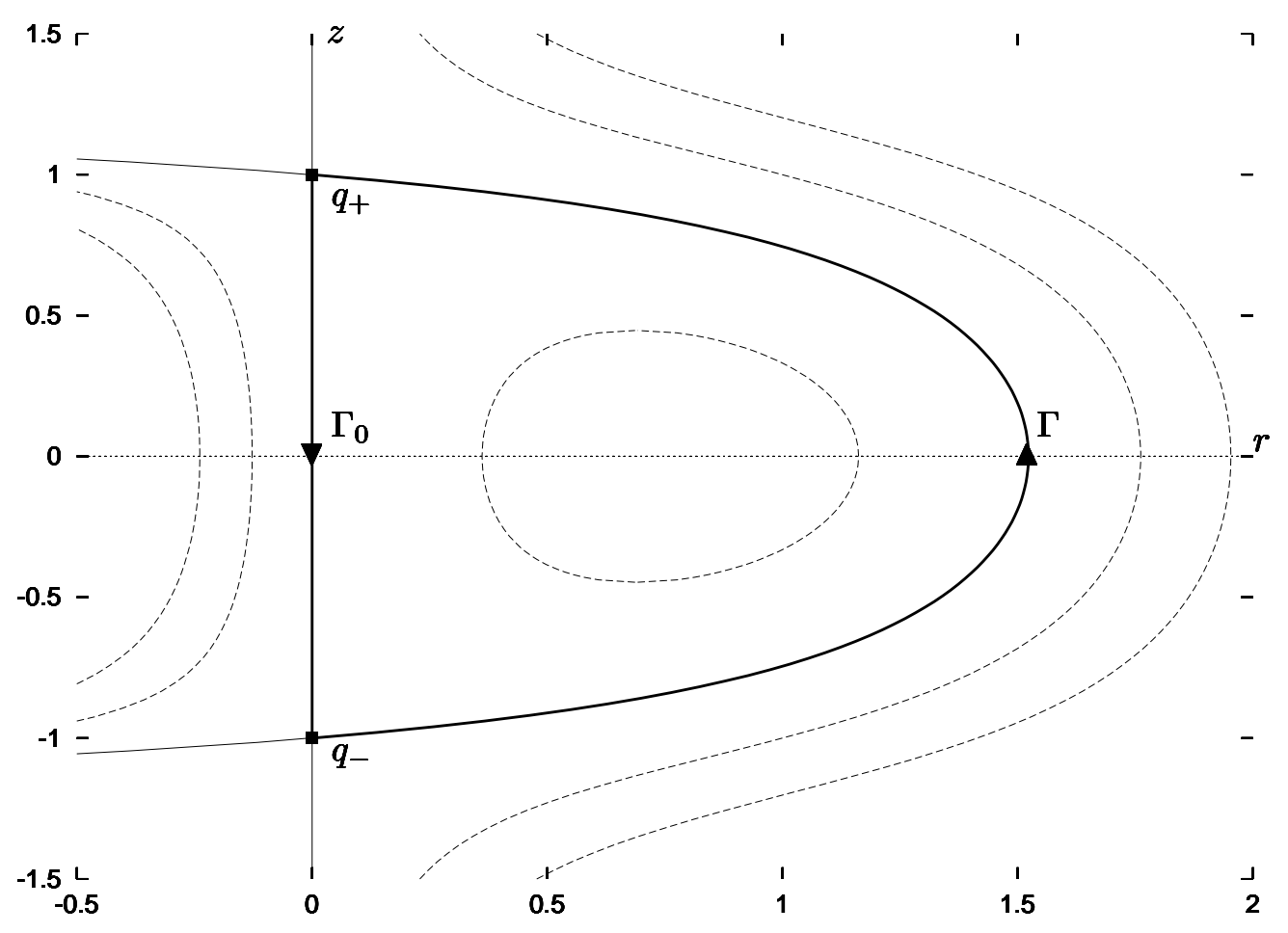

FIG. 2. The phase portrait of the area-preserving map (3) for $h=2$. The solid squares denote the hyperbolic fixed points $q_{ \pm}$. The thick lines denote the heteroclinic connections $\Gamma_{0}$ and $\Gamma$. The arrows denote the dynamics of the map on the connections

and the area-preserving map

$$
g(r, z)=\left(\phi\left(r+\phi^{-1}(z)\right)-z, r+\phi^{-1}(z)\right)
$$

where $\phi^{-1}(z)=(c z-s) /(c-s z)=-\phi(-z)$ is the inverse transformation of $(2)$. The phase portrait of this map is sketched in figure 2 . Its main dynamical properties are described in the next lemma.

LEMMA 2. The area-preserving map (3) verifies the following properties:

(i) The points $q_{ \pm}=(0, \pm 1)$ are hyperbolic fixed points of $g$ and

$$
\operatorname{spec}\left[D g\left(q_{ \pm}\right)\right]=\left\{\mathrm{e}^{h}, \mathrm{e}^{-h}\right\} \text {. }
$$

(ii) The function $J(r, z)=\left(c^{2}-s^{2} z^{2}\right) r^{2}+2 c s\left(z^{2}-1\right) r$ is a first integral and the level $J^{-1}(0)$ contains two heteroclinic connections between the hyperbolic fixed points.

(iii) These heteroclinic connections are $\Gamma_{0}=\left\{(r, z) \in \mathbb{R}^{2}: r=0,|z|<1\right\}$ and

$$
\Gamma=\left\{(r, z) \in \mathbb{R}^{2}: r=\phi(z)-\phi^{-1}(z)=\frac{2 c s\left(1-z^{2}\right)}{c^{2}-s^{2} z^{2}},|z|<1\right\} .
$$

(iv) The diffeomorphism $\gamma=(r, z): \mathbb{R} \rightarrow \Gamma, z(t)=\tanh (t / 2), r(t)=z(t+h)-$ $z(t-h)$, is a natural parametrization of the connection $\Gamma$; that is, $g(\gamma(t))=\gamma(t+h)$.

(v) The map $g$ is $R$-reversible and $R(\gamma(t))=\gamma(-t)$, where $R(r, z)=(r,-z)$.

Proof. It is a direct computation, so it is more enlightening to explain how these formulae are guessed. The canonical change of variables $(r, z) \mapsto\left(z, w=r+\phi^{-1}(z)\right)$ 
transforms (3) into the planar standard-like map

$$
\bar{g}(z, w)=(w, \psi(w)-z), \quad \psi(w)=\phi(w)+\phi^{-1}(w)=\frac{2 w}{c^{2}-s^{2} w^{2}}
$$

introduced by McMillan, which has similar properties [26, page 232]. For instance,

$$
\bar{J}(z, w)=s^{2}\left(z^{2}-1\right)\left(w^{2}-1\right)-(z-w)^{2}
$$

is a known first integral of the McMillan map and its zero-level set $\bar{J}^{-1}(0)$ contains two heteroclinic connections between the hyperbolic fixed points $\bar{q}_{-}=(-1,-1)$ and $\bar{q}_{+}=(1,1)$. From the relation $\psi(z)=\phi(z)+\phi^{-1}(z)$, we get that

$$
\bar{g}^{k}\left(z, \phi^{ \pm 1}(z)\right)=\left(\phi^{ \pm k}(z), \phi^{ \pm(k+1)}(z)\right)
$$

for all $k \in \mathbb{Z}$. Thus, using that $\phi:(-1,1) \rightarrow(-1,1)$ is a diffeomorphism such that $\lim _{k \rightarrow \pm \infty} \phi^{k}(z)= \pm 1$ for all $z \in(-1,1)$, we see that the heteroclinic connections are

$$
\bar{\Gamma}_{0}=\left\{w=\phi^{-1}(z)\right\}, \quad \bar{\Gamma}=\{w=\phi(z)\} .
$$

The change $(z, w) \mapsto\left(r=w-\phi^{-1}(z), z\right)$ transforms $\bar{\Gamma}_{0}$ into $\Gamma_{0}=\{r=0\}$ and $\bar{\Gamma}$ into $\Gamma=\left\{r=\phi(z)-\phi^{-1}(z)\right\}$. Finally, the natural parametrization follows from the relations $\phi(z(t))=z(t+h)$ and $r(t)=\phi(z(t))-\phi^{-1}(z(t))=z(t+h)-z(t-h)$.

Next, we construct a volume-preserving map using the area-preserving map (3). The methodology consists, roughly speaking, in "to rotate" the right half-plane $\{r>$ $0\}$ of figure 2 around the vertical axis, using "canonical" cylindrical coordinates [22]. The map becomes fully three-dimensional if we introduce any non-trivial dynamics in the cylindrical angular variable $\theta \in \mathbb{T}:=\mathbb{R} / 2 \pi \mathbb{Z}$. For instance, a rigid rotation $\theta \mapsto \Theta=\theta+\omega$ suffices. See also remark 2. The surface of revolution $\Sigma$ obtained from the curve $\Gamma$ is the bi-dimensional heteroclinic connection we were looking for.

The construction would be a little obscure if we use directly the Cartesian coordinates $(x, y, z)$. Hence, as an intermediate step, it is convenient to introduce the cylindrical angle $\theta \in \mathbb{T}$ and the cylindrical radius $\sqrt{2 r}>0$. That is, we will work with the "canonical" cylindrical coordinates $(r, \theta, z)$ defined by the relations

$$
x=\sqrt{2 r} \cos \theta, \quad y=\sqrt{2 r} \sin \theta, \quad z=z .
$$

The term "canonical" means that $\mathrm{d} x \wedge \mathrm{d} y \wedge \mathrm{d} z=\mathrm{d} r \wedge \mathrm{d} \theta \wedge \mathrm{d} z$. Consider the map $(r, \theta, z) \mapsto(R, \Theta, Z)$, given by

$$
\Theta=\theta+\omega, \quad(R, Z)=g(r, z)
$$

where $g$ is the area-preserving map (3). This map preserves volume, since

$$
\mathrm{d} X \wedge \mathrm{d} Y \wedge \mathrm{d} Z=-\mathrm{d} R \wedge \mathrm{d} Z \wedge \mathrm{d} \Theta=-\mathrm{d} r \wedge \mathrm{d} z \wedge \mathrm{d} \theta=\mathrm{d} x \wedge \mathrm{d} y \wedge \mathrm{d} z .
$$

Let

$$
\rho(r, z)=\left\{\begin{array}{cc}
\sqrt{\left(\phi\left(r+\phi^{-1}(z)\right)-z\right) / r}, & r \neq 0, \\
\sqrt{\phi^{\prime}\left(\phi^{-1}(z)\right)}, & r=0 .
\end{array}\right.
$$


This function $\rho(r, z)$ is analytic at $r=0$ for $|z|<c / s$. Using coordinates (4) in the map defined by (5), we get that, in Cartesian coordinates, the map that we want is

$$
\left(\begin{array}{l}
X \\
Y \\
Z
\end{array}\right)=f\left(\begin{array}{l}
x \\
y \\
z
\end{array}\right)=\left(\begin{array}{ccc}
\cos \omega & -\sin \omega & 0 \\
\sin \omega & \cos \omega & 0 \\
0 & 0 & 1
\end{array}\right)\left(\begin{array}{c}
\rho(r, z) x \\
\rho(r, z) y \\
r+\phi^{-1}(z)
\end{array}\right)
$$

where $r=\left(x^{2}+y^{2}\right) / 2$. We check, using formulation (6), that the map is well-defined and analytic on $\left\{(0,0, z) \in \mathbb{R}^{3}:|z|<c / s\right\}$. This was not immediately clear from (4), since the change to cylindrical coordinates is singular at $r=0$.

The map (6) is our unperturbed volume-preserving model. It depends on the characteristic exponent $h>0$ and the frequency $\omega \in \mathbb{T}$. The characteristic exponent measures the hyperbolicity of the problem. In particular, numerical computations or analytical studies about separatrix splittings for small values of $h$ will be hard, due to their exponentially smallness. For instance, we have only been able to prove a conjecture presented in $\S 7$ for $h \geq \log 16-\log (\sqrt{113}-9) \approx 2.282$.

The main dynamical properties of the integrable volume-preserving map (6) are described in the following lemma.

LEMMA 3. The volume-preserving map (6) verifies the following properties:

(i) The points $p_{ \pm}=(0,0, \pm 1)$ are hyperbolic fixed points of $f$ such that

$$
\operatorname{spec}\left[D f\left(p_{ \pm}\right)\right]=\left\{\mathrm{e}^{ \pm 2 h}, \mathrm{e}^{\mp h+\mathrm{i} \omega}, \mathrm{e}^{\mp h-\mathrm{i} \omega}\right\} .
$$

(ii) The function $I(x, y, z)=J\left(\left(x^{2}+y^{2}\right) / 2, z\right)$ is a first integral of $f$ and the level $I^{-1}(0)$ contains two heteroclinic connections between the hyperbolic fixed points.

(iii) The heteroclinic connections are $\Sigma_{0}=\left\{(0,0, z) \in \mathbb{R}^{3}:|z|<1\right\}$ and

$$
\Sigma=\left\{(x, y, z) \in \mathbb{R}^{3}: x^{2}+y^{2}=\frac{4 c s\left(1-z^{2}\right)}{c^{2}-s^{2} z^{2}},|z|<1\right\} .
$$

(iv) The diffeomorphism $\sigma: \mathbb{T} \times \mathbb{R} \rightarrow \Sigma, \sigma(\theta, t)=(x(\theta, t), y(\theta, t), z(t))$, given by

$$
\begin{aligned}
z(t) & =\tanh (t / 2) \\
r(t) & =z(t+h)-z(t-h) \\
x(\theta, t) & =\sqrt{2 r(t)} \cos \theta \\
y(\theta, t) & =\sqrt{2 r(t)} \sin \theta
\end{aligned}
$$

is a natural parametrization of $\Sigma$; that is, $f(\sigma(\theta, t))=\sigma(\theta+\omega, t+h)$.

(v) The map $f$ has the linear symmetry $S(x, y, z)=(-x,-y, z)$ and the involutive linear reversors $R(x, y, z)=(x,-y,-z)$ and $T(x, y, z)=(-x, y,-z)$. Besides, $S(\sigma(\theta, t))=\sigma(\theta+\pi, t), R(\sigma(\theta, t))=\sigma(-\theta,-t)$, and $T(\sigma(\theta, t))=\sigma(\pi-\theta,-t)$.

Proof. In the cylindrical coordinates $(r, \theta, z)$, the map $f$ acts in the form described in (5). Therefore, these properties follow directly from the properties of the map $g$ described in lemma 2 and the fact that the involutions $\theta \mapsto-\theta$ and $\theta \mapsto \pi-\theta$ are reversors of the rigid rotations $\theta \mapsto \theta+\omega$.

Remark 2. We could have considered that the frequency is not constant, but it depends on the first integral: $\omega=\omega(I)$. In that case, since the expression of the Melnikov function only needs the values of the dynamics on the saddle connection $\Sigma$, only the value $\omega_{0}=\omega(0)$ appears in the Melnikov computations.

The fixed sets of the reversors $R$ and $T$ are smooth curves. In fact, Fix $R$ is the $x$-axis and Fix $T$ is the $y$-axis. Besides, each fixed set intersects transversely the 
saddle connection at a couple of opposite points, namely

$$
\Sigma \cap \operatorname{Fix} R=\left\{\xi^{+}, \xi^{-}\right\}, \quad \Sigma \cap \operatorname{Fix} T=\left\{\zeta^{+}, \zeta^{-}\right\}
$$

where $\xi^{ \pm}=( \pm \eta, 0,0), \zeta^{ \pm}=(0, \pm \eta, 0)$, and $\eta=2 \sqrt{s / c}$. Besides, $\xi^{+}=\sigma(0,0)$, $\xi^{-}=\sigma(\pi, 0), \zeta^{+}=\sigma(\pi / 2,0)$, and $\zeta^{-}=\sigma(3 \pi / 2,0)$. The question about when these symmetric heteroclinic points persist is answered in the following proposition.

Proposition 4. Let $S, R$ and $T$ be the symmetry and the reversors introduced in lemma 3. Let $\xi^{ \pm}$and $\zeta^{ \pm}$be the symmetric heteroclinic points of the map (6) on the $x$-axis and $y$-axis, respectively. Let $P_{\epsilon}$ be the set of primary heteroclinic intersections of the perturbed map $f_{\epsilon}=p_{\epsilon} \circ f$, where $p_{\epsilon}=\mathrm{Id}+\epsilon \kappa$ and $\kappa(x, y, z)=(0, \alpha(x), \beta(x, y))$.

(i) If $\alpha(x)$ is odd and $\beta(x, y)$ is even, then $f_{\epsilon}$ is $S$-symmetric and $S\left(P_{\epsilon}\right)=P_{\epsilon}$.

(ii) If $\beta(x, y)$ is even in $y$, then $f_{\epsilon} \circ R_{\epsilon}-R_{\epsilon} \circ f_{\epsilon}^{-1}=\mathrm{O}\left(\epsilon^{2}\right)$, where $R_{\epsilon}=p_{\epsilon} \circ R$. If, in addition, $\alpha(0)=0$ and $\beta(0, \alpha(x))=0$, then $f_{\epsilon}$ is $R_{\epsilon}$-reversible, $R_{\epsilon}\left(P_{\epsilon}\right)=P_{\epsilon}$, and there exists points $\xi_{\epsilon}^{ \pm}=\xi^{ \pm}+\mathrm{O}(\epsilon) \in P_{\epsilon} \cap$ Fix $R_{\epsilon}$.

(iii) If $\alpha(x)$ is odd and $\beta(x, y)$ is even in $x$, then $f_{\epsilon} \circ T_{\epsilon}-T_{\epsilon} \circ f_{\epsilon}^{-1}=\mathrm{O}\left(\epsilon^{2}\right)$, where $T_{\epsilon}=p_{\epsilon} \circ T$. If, in addition, $\alpha(0)=0$ and $\beta(0, \alpha(x))=0$, then $f_{\epsilon}$ is $T_{\epsilon}$-reversible, $T_{\epsilon}\left(P_{\epsilon}\right)=P_{\epsilon}$, and there exists points $\zeta_{\epsilon}^{ \pm}=\zeta^{ \pm}+\mathrm{O}(\epsilon) \in P_{\epsilon} \cap$ Fix $T_{\epsilon}$.

Proof. (i) If $\alpha(x)$ is odd and $\beta(x, y)$ is even, then $\kappa \circ S=S \circ \kappa$ and

$$
p_{\epsilon} \circ S=(\operatorname{Id}+\epsilon \kappa) \circ S=S+\epsilon S \circ \kappa=S \circ(\operatorname{Id}+\epsilon \kappa)=S \circ p_{\epsilon} .
$$

Therefore, $f_{\epsilon} \circ S=p_{\epsilon} \circ f \circ S=p_{\epsilon} \circ S \circ f=S \circ p_{\epsilon} \circ f=S \circ f_{\epsilon}$.

(ii) If $\beta(x, y)$ is even in $y$, then $\kappa \circ R=-R \circ \kappa$. Besides, $p_{\epsilon}^{-1}=\mathrm{Id}-\epsilon \kappa+\mathrm{O}\left(\epsilon^{2}\right)$. Therefore, $R_{\epsilon}=(\mathrm{Id}+\epsilon \kappa) \circ R=R-\epsilon R \circ \kappa=R \circ(\mathrm{Id}-\epsilon \kappa)=R \circ p_{\epsilon}^{-1}+\mathrm{O}\left(\epsilon^{2}\right)$ and $f_{\epsilon} \circ R_{\epsilon}=p_{\epsilon} \circ f \circ R \circ p_{\epsilon}^{-1}+\mathrm{O}\left(\epsilon^{2}\right)=p_{\epsilon} \circ R \circ f^{-1} \circ p_{\epsilon}^{-1}+\mathrm{O}\left(\epsilon^{2}\right)=R_{\epsilon} \circ f_{\epsilon}^{-1}+\mathrm{O}\left(\epsilon^{2}\right)$.

If $\alpha(0)=0$ and $\beta(0, \alpha(x))=0$, then $\kappa^{2}(x, y, z)=(0, \alpha(0), \beta(0, \alpha(x)))=0$ and $p_{\epsilon}^{-1}=\mathrm{Id}-\epsilon \kappa$, so all the $\mathrm{O}\left(\epsilon^{2}\right)$ terms above vanish.

(iii) If $\alpha(x)$ is odd and $\beta(x, y)$ is even in $x$, then $\kappa \circ T=-T \circ \kappa$, so it suffices to replace $R$ with $T$ in the previous item.

When $R_{\epsilon}$ and $T_{\epsilon}$ are true reversors, their fixed sets are

$$
\begin{aligned}
\operatorname{Fix} R_{\epsilon} & =\left\{(x, y, z) \in \mathbb{R}^{3}: y=\epsilon \alpha(x) / 2, z=\epsilon \beta(x, \epsilon \alpha(x) / 2) / 2\right\}, \\
\operatorname{Fix} T_{\epsilon} & =\left\{(x, y, z) \in \mathbb{R}^{3}: x=0, z=\epsilon \beta(0, y) / 2\right\}
\end{aligned}
$$

which are $\mathrm{O}(\epsilon)$-close to the $x$-axis and $y$-axis. (We have used that $\alpha(0)=0$.)

4. The Melnikov function. Next, we want to derive an explicit expression for the Melnikov function associated to the volume-preserving perturbations

$$
f_{\epsilon}=(\mathrm{Id}+\epsilon \kappa) \circ f, \quad \kappa(x, y, z)=(0, \alpha(x), \beta(x, y)) .
$$

Other perturbations can also be studied. We do not aspire to be exhaustive.

If $\alpha(0)=\beta(0,0)=0$, then $f_{\epsilon}(0,0, z)=f(0,0, z)$ and the one-dimensional heteroclinic connection $\Sigma_{0}$ is preserved under the perturbation (8). There is no similar persistence result for the two-dimensional heteroclinic connection $\Sigma$.

The first integral given in lemma 3 is $I(x, y, z)=J(r, z)$, where $r=\left(x^{2}+y^{2}\right) / 2$ and $J(r, z)=\left(c^{2}-s^{2} z^{2}\right) r^{2}+2 c s\left(z^{2}-1\right) r$. Besides, $r=2 c s\left(1-z^{2}\right) /\left(c^{2}-s^{2} z^{2}\right)$ on the saddle connection $\Sigma$. Finally, if the perturbation has the form (8), then the Melnikov function (1) can be written as

$$
M: \Sigma \rightarrow \mathbb{R}, \quad M(x, y, z)=\sum_{k \in \mathbb{Z}} m\left(x_{k}, y_{k}, z_{k}\right)
$$


where $\left(x_{k}, y_{k}, z_{k}\right)=f^{k}(x, y, z)$ and

$$
\begin{aligned}
m(x, y, z) & =\langle\nabla I(x, y, z), \kappa(x, y, z)\rangle \\
& =\partial_{y} I(x, y, z) \alpha(x)+\partial_{z} I(x, y, z) \beta(x, y) \\
& =y \partial_{r} J(r, z) \alpha(x)+\partial_{z} J(r, z) \beta(x, y) \\
& =2 \operatorname{cs} y\left(1-z^{2}\right) \alpha(x)+2 s z r(2 c-s r) \beta(x, y) .
\end{aligned}
$$

On the other hand, the natural parametrization $\sigma=(x, y, z): \mathbb{T} \times \mathbb{R} \rightarrow \Sigma$ given in (7) provides a diffeomorphism between the saddle connection $\Sigma$ an the cylinder $\mathbb{T} \times \mathbb{R}$, so that objects defined over $\Sigma$ can be considered as depending on an angular variable $\theta \in \mathbb{T}$ and a hyperbolic variable $t \in \mathbb{R}$. Henceforth, we will abuse the notation by not giving these objects new names. Thus, the Melnikov function (9) becomes

$$
M: \mathbb{T} \times \mathbb{R} \rightarrow \mathbb{R}, \quad M(\theta, t)=\sum_{k \in \mathbb{Z}} m(\theta+k \omega, t+k h)
$$

where

$$
\begin{aligned}
m(\theta, t) & =\lambda(t) y(\theta, t) \alpha(x(\theta, t))+\mu(t) \beta(x(\theta, t), y(\theta, t)) \\
& =\rho(t) \lambda(t) \alpha(\rho(t) \cos \theta) \sin \theta+\mu(t) \beta(\rho(t) \cos \theta, \rho(t) \sin \theta)
\end{aligned}
$$

and

$$
\begin{aligned}
& r(t)=z(t+h)-z(t-h)=\frac{2 c s}{\cosh ((t+h) / 2) \cosh ((t-h) / 2)}, \\
& \rho(t)=\sqrt{2 r(t)} \\
& \lambda(t)=2 c s\left(1-z(t)^{2}\right)=4 c s z^{\prime}(t)=\frac{2 c s}{\cosh ^{2}(t / 2)}, \\
& \mu(t)=2 s z(t) r(t)(2 c-s r(t))=-4 c s r^{\prime}(t) .
\end{aligned}
$$

The rest of the paper deals with the computation and description of the zero-level set

$$
Z=M^{-1}(0)=\{(\theta, t) \in \mathbb{T} \times \mathbb{R}: M(\theta, t)=0\}
$$

for several simple perturbations (8). In order to make easier that, we recall that the Melnikov function was invariant by the unperturbed map. In the current context, this implies that the Melnikov function $M$ satisfies

$$
M(\theta+\omega, t+h)=M(\theta, t)=M(\theta+2 \pi, t)
$$

and therefore the zero-set $Z$ is $(2 \pi, 0)$ and $(\omega, h)$-periodic. This is, if $\left(\theta^{*}, t^{*}\right) \in Z$ then $\left(\theta^{*}+\omega, t^{*}+h\right) \in Z$ and $\left(\theta^{*}+2 \pi, t^{*}\right) \in Z$.

A tilde will always denote the projection of a periodic object to the quotient torus

$$
\tilde{\tau}(\omega, h)=\tilde{\tau}:=(\mathbb{T} \times \mathbb{R}) /(\omega, h) \mathbb{Z}=\mathbb{R}^{2} /((2 \pi, 0) \mathbb{Z}+(\omega, h) \mathbb{Z})
$$

which is diffeomorphic to the quotient of the saddle connection by the unperturbed map. The study of the projected set $\tilde{Z}=\tilde{M}^{-1}(0) \subset \tilde{\tau}$ is easier, because $\tilde{\tau}$ is compact.

The torus is represented in figure 3 as the rectangle $[0,2 \pi] \times[0, h]$ with the appropriate identifications. We have not chosen the parallelogram shown in thin lines in 


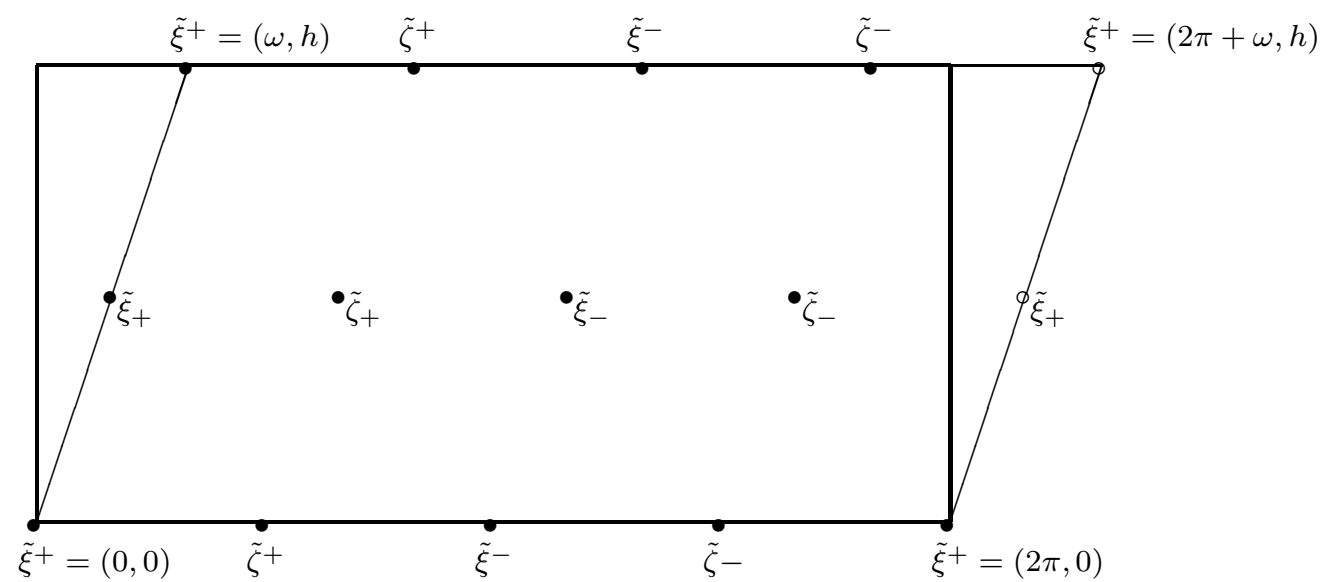

FIG. 3. A rectangular representation of the torus $\tilde{\tau}$ and the symmetric points described in lemma 5 for $\omega=\pi / 3$. Opposites sides of the rectangle are identified, although the identification of the horizontal ones is shifted by an amount equal to $\omega$.

that figure as the representation of the torus, because its shape changes in $\omega$, hindering posterior comparisons and the study of bifurcations that take place in $\omega$.

Remark 3. Sometimes we will restrict ourselves to the case $\omega=0$, which is called no-swirl in fluid dynamics. This is the simplest one, because then the quotient torus is a product: $\tilde{\tau}(0, h)=(\mathbb{R} / 2 \pi \mathbb{Z}) \times(\mathbb{R} / h \mathbb{Z})$, and the variable $t$ is defined modulo $h$. Although the unperturbed map has just a bi-dimensional dynamics for $\omega=0$, it is still an interesting case - the perturbation will create a real three-dimensional dynamics.

Let us check that $\tilde{Z}$ contains at least eight symmetric points and has some useful symmetries and more periodicities when the perturbation preserves the symmetry and reversors of the unperturbed map. The symmetric points are shown in figure 3 .

LEMma 5. Let $\tilde{Z}$ be the projection onto the torus (14) of the zero-level set of the Melnikov function (10).

(i) If $\alpha(x)$ is odd and $\beta(x, y)$ is even, then $\tilde{Z}$ is $(\pi, 0)$-periodic: $\tilde{Z}=\tilde{Z}+(\pi, 0)$.

(ii) If $\alpha(x)$ is even and $\beta(x, y)$ is odd, then $\tilde{Z}$ is $(\pi, 0)$-periodic: $\tilde{Z}=\tilde{Z}+(\pi, 0)$.

(iii) If $\beta(x, y)$ is even in $y$, then $\tilde{Z}$ contains (and is symmetric with regard to) the points $\tilde{\xi}^{+}=(0,0), \tilde{\xi}^{-}=(\pi, 0), \tilde{\xi}_{+}=(\omega / 2, h / 2)$, and $\tilde{\xi}_{-}=(\pi+\omega / 2, h / 2)$.

(iv) If $\alpha(x)$ is odd and $\beta(x, y)$ is even in $x$, then $\tilde{Z}$ contains (and is symmetric with regard to $)$ the points $\tilde{\zeta}^{+}=(\pi / 2,0), \tilde{\zeta}^{-}=(3 \pi / 2,0), \tilde{\zeta}_{+}=(\pi / 2+\omega / 2, h / 2)$, and $\tilde{\zeta}_{-}=(3 \pi / 2+\omega / 2, h / 2)$.

Proof. We could write a geometric proof based on the geometric properties established in proposition 4 , but instead, we give a shorter analytic proof.

We consider the Melnikov function $M$ as a function defined on the plane $\mathbb{R}^{2}$ with periods $(2 \pi, 0)$ and $(\omega, h)$. Assume that $M$ is odd with regard to a point $\left(\theta_{0}, t_{0}\right) \in \mathbb{R}^{2}$. Then $M^{-1}(0)$ contains (and is symmetric with regard to) the point $\left(\theta_{0}, t_{0}\right)$. But $M^{-1}(0)$ also contains (and is symmetric with regard to) the points $\left(\theta_{0}+p, t_{0}+q\right)$ for any semi-period $(p, q)$ of $M$, because

$$
M\left(\theta_{0}+p, t_{0}+q\right)=-M\left(\theta_{0}-p, t_{0}-q\right)=-M\left(\theta_{0}+p, t_{0}+q\right) .
$$

Hence, it suffices to check that:

(i) If $\alpha(x)$ is odd and $\beta(x, y)$ is even, then $M(\theta, t)$ is $\pi$-periodic in $\theta$;

(ii) If $\alpha(x)$ is even and $\beta(x, y)$ is odd, then $M(\theta, t)$ is $\pi$-antiperiodic in $\theta$; 
(iii) If $\beta(x, y)$ is even in $y$, then $M(\theta, t)$ is odd with regard to $(0,0)$; and

(iv) If $\alpha(x)$ is odd and $\beta(x, y)$ is even in $x, M(\theta, t)$ is odd with regard to $(\pi / 2,0)$. The functions $r(t), \rho(t)$, and $\lambda(t)$ given in (12) are even, whereas $\mu(t)$ is odd. Hence, the function (11) and the Melnikov function (10) verify (i)-(iv).

Remark 4. One can obtain more symmetries or periodicities under more restrictive hypotheses. For instance, using basic trigonometric properties, one checks that $Z$ is $(\pi / 2,0)$-periodic when $\kappa(x, y, z)=(0, x, 0)$, or when $\kappa(x, y, z)=(0,0, \beta(x, y))$ for some $\beta(x, y)$ such that $\beta(x, y)=\beta(-y, x)$ or $\beta(x, y)=-\beta(-y, x)$.

Remark 5. It turns out that $\tilde{Z} \neq \emptyset$, even if the volume-preserving perturbation (8) has no symmetries. This has to do with the existence of an area form $\tilde{\eta}$ over the torus $\tilde{\tau}$ such that the integral of the two-form $\tilde{M} \tilde{\eta}$ vanishes. Therefore, the Melnikov function has to be zero at some points. This idea was used in [23]. We skip the details.

5. Bounds on the complexity of the primary heteroclinic set. First, we shall establish an upper bound on the cardinality of the horizontal sections of the zero-level set $Z$ under polynomial perturbations of the form (8). These horizontal sections are defined as

$$
Z_{t_{0}}=\left\{\theta \in \mathbb{T}: M\left(\theta, t_{0}\right)=0\right\}=\left\{\theta \in \mathbb{T}:\left(\theta, t_{0}\right) \in Z\right\}, \quad t_{0} \in \mathbb{R}
$$

Proposition 6. If $\alpha(x) \in \mathbb{R}_{n-1}[x]$ and $\beta(x, y) \in \mathbb{R}_{n}[x, y]$ for some integer $n \geq 1$, then either $Z_{t_{0}}=\mathbb{T}$ or $\# Z_{t_{0}} \leq 2 n$.

Proof. The function $m(\theta, t)$ given in (11) has the following simple forms under monomial perturbations. If $\alpha(x)=x^{i-1}$ and $\beta(x, y)=0$, then $m(\theta, t)=$ $(2 r(t))^{i / 2} \lambda(t) \cos ^{i-1} \theta \sin \theta$, whereas if $\alpha(x)=0$ and $\beta(x, y)=x^{i} y^{j}$, then $m(\theta, t)=$ $(2 r(t))^{(i+j) / 2} \mu(t) \cos ^{i} \theta \sin ^{j} \theta$. Therefore, the Fourier expansion of $m(\theta, t)$ when $\alpha(x) \in$ $\mathbb{R}_{n-1}[x]$ and $\beta(x, y) \in \mathbb{R}_{n}[x, y]$ has only the central $2 n+1$ harmonics. That is, $m(\theta, t)=\sum_{|j| \leq n} m_{j}(t) \mathrm{e}^{\mathrm{i} j \theta}$ for some coefficients $m_{j}(t)$. Thus the Fourier expansion of the Melnikov function (10) has the same form, since

$$
\begin{aligned}
M(\theta, t) & =\sum_{k \in \mathbb{Z}} m(\theta+k \omega, t+k h) \\
& =\sum_{|j| \leq n} \sum_{k \in \mathbb{Z}} m_{j}(t+k h) \mathrm{e}^{\mathrm{i} j(\theta+k \omega)} \\
& =\sum_{|j| \leq n} M_{j}(t) \mathrm{e}^{\mathrm{i} j \theta}
\end{aligned}
$$

where $M_{j}(t)=\sum_{k \in \mathbb{Z}} \mathrm{e}^{\mathrm{i} j k \omega} m_{j}(t+k h)$. To end the proof, it suffices to note that any non-zero trigonometric polynomial like $M_{t_{0}}(\theta):=M\left(\theta, t_{0}\right)=\sum_{|j| \leq n} M_{j}\left(t_{0}\right) \mathrm{e}^{\mathrm{i} j \theta}$ has at most $2 n$ different roots in $\mathbb{T}$.

If $\omega=0$, there exists a similar bound for the cardinal of the vertical sections. This new bound is obtained by using some elementary facts of the theory of elliptic functions. We recall that a function is elliptic when it is meromorphic in the whole complex plane and has two complex periods that are independent over the reals. The order of a non-constant elliptic function is the number of its poles (or zeros), counted with multiplicity, that lie in a cell. A cell of an elliptic function with periods $p_{1}$ and $p_{2}$ is a parallelogram with vertexes $s, s+p_{1}, s+p_{1}+p_{2}$, and $s+p_{2}$ such that its sides do not contain neither zeros nor poles. For a general background on elliptic functions, we refer to [33]. 
We realized in remark 3 that the Melnikov function $M(\theta, t)$ is $h$-periodic in the vertical coordinate $t$ when the frequency $\omega$ is zero. In that case, the vertical sections of the projected zero-level set $\tilde{Z}=\tilde{M}^{-1}(0) \subset \tilde{\tau}$ defined as

$$
\tilde{Z}^{\theta_{0}}=\left\{t \in \mathbb{R} / h \mathbb{Z}: \tilde{M}\left(\theta_{0}, t\right)=0\right\}=\left\{t \in \mathbb{R} / h \mathbb{Z}:\left(\theta_{0}, t\right) \in \tilde{Z}\right\}
$$

are subsets of the quotient space $\mathbb{R} / h \mathbb{Z}$.

Proposition 7. Assume that the perturbation (8) is polynomial and $\omega=0$. Let $\tilde{Z}^{\theta_{0}}$ be any vertical section which does not cover the whole set $\mathbb{R} / h \mathbb{Z}$. Let $l, n \in \mathbb{N}$.

(i) If $\alpha(x) \in \mathbb{R}_{2 l-1}[x]$ is odd and $\beta(x, y)=0$, then $\# \tilde{Z}^{\theta_{0}} \leq l$.

(ii) If $\alpha(x)=0$ and $\beta(x, y) \in \mathbb{R}_{2 n}[x, y]$ is even, then $\# \tilde{Z}^{\theta_{0}} \leq n+1$.

(iii) If $\alpha(x) \in \mathbb{R}_{2 l-1}[x]$ is odd and $\beta(x, y) \in \mathbb{R}_{2 n}[x, y]$ is even, then $\# \tilde{Z}^{\theta_{0}} \leq$ $\max (l, n+2)$.

Proof. These three items a based on the following formulae. If $\alpha(x)=x^{2 j-1}$ and $\beta(x, y)=0$, then $m(\theta, t)=(2 r(t))^{j} \lambda(t) \cos ^{2 j-1} \theta \sin \theta$. If $\alpha(x)=0$ and $\beta(x, y)=$ $x^{i} y^{2 j-i}$, then $m(\theta, t)=(2 r(t))^{j} \mu(t) \cos ^{i} \theta \sin ^{2 j-i} \theta$. Hence, if the polynomial $\alpha(x) \in$ $\mathbb{R}_{2 l-1}[x]$ is odd and the polynomial $\beta(x, y) \in \mathbb{R}_{2 n}[x, y]$ is even, the function $m(\theta, t)$ has the form

$$
m(\theta, t)=\lambda(t) \sum_{j=1}^{l} a_{j}(\theta)(r(t))^{j}+\mu(t) \sum_{j=1}^{n} b_{j}(\theta)(r(t))^{j}
$$

for some trigonometric polynomials $a_{j}(\theta)$ and $b_{j}(\theta)$. Since $\omega=0$, we get that

$$
\tilde{M}^{\theta_{0}}(t):=\tilde{M}\left(\theta_{0}, t\right)=\sum_{j=1}^{l} a_{j}\left(\theta_{0}\right) A_{j}(t)+\sum_{j=1}^{n} b_{j}\left(\theta_{0}\right) B_{j}(t)
$$

where $A_{j}(t)=\sum_{k \in \mathbb{Z}} \lambda(t+k h)(r(t+k h))^{j}$ and $B_{j}(t)=\sum_{k \in \mathbb{Z}} \mu(t+k h)(r(t+k h))^{j}$.

The functions $z(t), r(t), \lambda(t)$, and $\mu(t)$ are $2 \pi$ i-periodic and meromorphic in $\mathbb{C}$. On the one hand, the poles of $z(t)$ are the points in the set $\pi \mathrm{i}+2 \pi \mathrm{i} \mathbb{Z}$, all of them simple, and so $\lambda(t)=4 c s z^{\prime}(t)$ has the same poles, but they are double ones. On the other hand, the poles of $r(t)$ are the points in the sets $\pm h+\pi \mathrm{i}+2 \pi \mathrm{i} \mathbb{Z}$, all of them simple, and so $\mu(t)=-4 c s r^{\prime}(t)$ has the same poles, but they are double ones.

Thus, $A_{j}(t), B_{j}(t)$, and $\tilde{M}^{\theta_{0}}(t)$ are elliptic functions with periods $h$ and $2 \pi \mathrm{i}$. Their poles are the points in the set $\pi \mathrm{i}+h \mathbb{Z}+2 \pi \mathrm{i} \mathbb{Z}$. Their orders are at $\operatorname{most} \max (j, 2)$, $j+2$, and $\max (l, n+2)$, respectively. To end the common part of the proof, we note that $\tilde{M}^{\theta_{0}}(t)$ is non-constant, because $\tilde{Z}^{\theta_{0}} \neq \mathbb{R} / h \mathbb{Z}$.

(i) If $\beta(x, y)=0$, the elliptic function (15) becomes $\tilde{M}^{\theta_{0}}(t)=\sum_{j=1}^{l} a_{j}\left(\theta_{0}\right) A_{j}(t)$ and its order is at most $\max (l, 2)$, so that it has at $\operatorname{most} \max (l, 2)$ roots in a cell and $\# \tilde{Z}^{\theta_{0}} \leq \max (l, 2)$. We can substitute this last bound by $\# \tilde{Z}^{\theta_{0}} \leq l$ because if $\alpha(x)=x$ and $\omega=0$, then either $\tilde{Z}^{\theta_{0}}=\mathbb{R} / h \mathbb{Z}$ or $\tilde{Z}^{\theta_{0}}=\emptyset$, see item (iv) of theorem 17 .

(ii) If $\alpha(x)=0$, the elliptic function (15) becomes $\tilde{M}^{\theta_{0}}(t)=\sum_{j=1}^{n} b_{j}\left(\theta_{0}\right) B_{j}(t)$ and is odd, because $\mu(t)$ is odd and $r(t)$ is even. Its order is at most $n+2$, but the rough bound $\# \tilde{Z}^{\theta_{0}} \leq n+2$ can be improved using the symmetry. We get that $\tilde{M}^{\theta_{0}}(h / 2+\pi \mathrm{i})=0$, because

$$
\tilde{M}^{\theta_{0}}(h / 2+\pi \mathrm{i})=\tilde{M}^{\theta_{0}}(-h / 2+\pi \mathrm{i})=\tilde{M}^{\theta_{0}}(-h / 2-\pi \mathrm{i})=-\tilde{M}^{\theta_{0}}(h / 2+\pi \mathrm{i}) .
$$

This means that $\tilde{M}^{\theta_{0}}(t)$ has at most $n+1$ real roots modulo $h$.

(iii) In this case, the bound $\# \tilde{Z}^{\theta_{0}} \leq \max (l, n+2)$ can not be improved. 
Remark 6. Proposition 6 also holds for the integrable trigonometric family of volume-preserving maps introduced in [22]. The proof does not requires any change. On the contrary, proposition 7 can not be directly translated into the trigonometric setting, because the complex singularities of the natural parametrization of the separatrix in that setting are more complicated.

Recall that in the introduction we had a brief discussion of the type of primary heteroclinic intersection that appear. In our case, it is enough to remember that primary intersections are the intersections that arise as the continuation of the nondegenerate zeroes of the Melnikov function.

Any vertical curve intersects the horizontal line $\left\{t=t_{0}\right\}$ in at least one point, so the number of vertical curves can not be larger than the cardinal of the horizontal sections of the zero-level set. Therefore, as a by-product of proposition 6 , we get that there are at most $2 n$ vertical curves when $\alpha(x) \in \mathbb{R}_{n-1}[x]$ and $\beta(x, y) \in \mathbb{R}_{n}[x, y]$. In fact, propositions 6 and 7 have stronger consequences on the homology/homotopy classes of the heteroclinic intersections of the invariant manifolds.

We recall that $\tilde{Z}$ is the projection of the zero-level set $Z=M^{-1}(0)$ onto the torus $\tilde{\tau}$ defined in (14). Assume that 0 is a regular value of the Melnikov function. Then $\tilde{Z}$ is a submanifold of the torus and its connected components are closed smooth curves. Therefore, once fixed an induced orientation on the torus, we can assign to each connected component $\tilde{\gamma}$ of $\tilde{Z}$ its homology class $[\tilde{\gamma}] \in H_{1}\left(\mathbb{T}^{2}\right)=\mathbb{Z}^{2}$.

In the case of the torus $\tilde{\tau}$, we will identify horizontal lines with the class $(1,0)$ and vertical lines, generated by the vector $(\omega, h)$, with the class $(0,1)$. Thus, $[\tilde{\gamma}]=$ $(p, q) \in \mathbb{Z}^{2}$ means that $\tilde{\gamma}$ is a closed curve that wraps around the torus $|p|$ times in the horizontal direction and $|q|$ times in the vertical one. For instance, the set $\tilde{Z}$ has four connected components with homology class $(0,1)$ or $(0,-1)$ in the subfigures $4(\mathrm{a})-$ $4(\mathrm{~d})$, whereas it has just two connected components with homology class $(1,-2)$ or $(-1,2)$ in the subfigures $4(\mathrm{f})-4(\mathrm{i})$. Subfigure $4(\mathrm{e})$ is excluded because then 0 is a singular value of the Melnikov function.

Remark 7 . With regard to the three types of heteroclinic curves mentioned in the introduction, we note that a connected component $\tilde{\gamma}$ such that $[\tilde{\gamma}]=(p, q)$ gives rise for $\epsilon$ small enough to vertical (resp., equatorial) (resp., bubble-type) curves when $q \neq 0$ (resp., $q=0$ but $p \neq 0$ ) (resp., $p=q=0$ ).

Remark 8. The first homology group and the first homotopy group (that is, the fundamental group) of a torus coincide: $H_{1}\left(\mathbb{T}^{2}\right)=\mathbb{Z}^{2}=\pi_{1}\left(\mathbb{T}^{2}\right)$. Hence, we could use homotopy instead of singular homology along this digression.

We need the following result from Morse theory.

LEMMA 8. If $a \in \mathbb{R}$ is a regular value of a smooth function $f: X \rightarrow \mathbb{R}$ defined over a compact manifold $X$, the homology class of the level set $L_{a}=f^{-1}(a)$ is trivial.

Proof. It suffices to prove this for Morse functions, because Morse functions are dense and the homology class of a closed curve does not change under small perturbations.

Let $a$ and $b$ be two regular values of $f$ such that $a<b$. Then $L_{a}$ and $L_{b}$ are the borders of the smooth manifold $f^{-1}([a, b])$, and so, they have the same homology class. Let $c$ be the maximum value of $f$. Since $f$ is Morse, there exists a unique point $x \in X$ such that $f(x)=c$. This point is a nondegenerate maximum, and so, if $\delta>0$ is small enough, $c-\delta$ is a regular value and $L_{c-\delta}$ is just a small closed curve around $x$. Hence, $L_{c-\delta}$ is contractible and its homology class is equal to zero.

The homology classes of the connected components of the projected zero-level set $\tilde{Z}$ are bounded in the following theorem. These bounds restrict the topological 
complexity of the primary heteroclinic set $P_{\epsilon}=Z+\mathrm{O}(\epsilon)$ for small values of $\epsilon$.

THEOREM 9. Assume that 0 is a regular value of the Melnikov function associated to the perturbation (8). Let $\tilde{\gamma}_{1}, \ldots, \tilde{\gamma}_{r}$ be connected components of the projected zerolevel set $\tilde{Z}=\tilde{M}^{-1}(0)$. Let $\left[\tilde{\gamma}_{1}\right]=\left(p_{1}, q_{1}\right), \ldots,\left[\tilde{\gamma}_{r}\right]=\left(p_{r}, q_{r}\right)$ be their homology classes.

(i) The homology of $\tilde{Z}$ is trivial: $\sum_{j}\left[\tilde{\gamma}_{j}\right]=\sum_{j}\left(p_{j}, q_{j}\right)=(0,0)$.

(ii) If $\alpha(x) \in \mathbb{R}_{n-1}[x]$ and $\beta(x, y) \in \mathbb{R}_{n}[x, y]$, then $2\left|q_{j}\right| \leq \sum_{j}\left|q_{j}\right| \leq 2 n$.

(iii) Assume that $\omega=0$. Let $l, n \in \mathbb{N}$. Then:

1. If $\alpha(x) \in \mathbb{R}_{2 l-1}[x]$ is odd and $\beta(x, y)=0$, then $2\left|p_{j}\right| \leq \sum_{j}\left|p_{j}\right| \leq l$.

2. If $\alpha(x)=0$ and $\beta(x, y) \in \mathbb{R}_{2 n}[x, y]$ is even, then $2\left|p_{j}\right| \leq \sum_{j}\left|p_{j}\right| \leq n+1$.

3. If $\alpha(x) \in \mathbb{R}_{2 l-1}[x]$ is odd and $\beta(x, y) \in \mathbb{R}_{2 n}[x, y]$ is even, then $2\left|p_{j}\right| \leq$ $\sum_{j}\left|p_{j}\right| \leq \max (l, n+2)$.

Proof. (i) It suffices to apply lemma 8 to the projected function $\tilde{M}: \mathbb{T}^{2} \rightarrow \mathbb{R}$.

(ii) We are under the hypotheses of proposition 6 and 0 is a regular value of the Melnikov function, so there exists some $t_{*} \in \mathbb{R}$ such that $\# Z_{t_{*}} \leq 2 n$. On the contrary, $Z_{t_{0}}=\mathbb{T}$ for all $t_{0} \in \mathbb{R}$, and the Melnikov function should be identically zero.

Using that $\tilde{Z}=\tilde{\gamma}_{1} \amalg \cdots \amalg \tilde{\gamma}_{r}$ and that each curve $\tilde{\gamma}_{j}$ wraps $\left|q_{j}\right|$ times in the vertical direction, we get that $\sum_{j}\left|q_{j}\right| \leq \sum_{j} \#\left(\tilde{\gamma}_{j} \cap\left(\mathbb{T} \times\left\{t_{*}\right\}\right)\right)=\# Z_{t_{*}} \leq 2 n$. Next, we obtain the bound $2\left|q_{j}\right|=\left|q_{j}\right|+\left|\sum_{i \neq j} q_{i}\right| \leq \sum_{i}\left|q_{i}\right| \leq 2 n$ from the identity $\sum_{j} q_{j}=0$.

(iii) It follows in a similar way, but from proposition 7 .

6. Splitting of separatrices. In this section we shall present two theorems about the splitting of our separatrix. In the first one, we shall establish a sufficient condition for the splitting of the separatrix under some entire perturbations, whereas in the second one we find a broad class of polynomial perturbations that split the separatrix. The sufficient condition is obtained through the study of the complex singularities of the Melnikov function. To be more precise, if the Melnikov function can be analytically extended for complex values of its variables and this extension has some nonremovable singularity, then the original Melnikov function can not be identically zero and the separatrix splits.

For simplicity, we have restricted our study to the perturbations of the form

$$
f_{\epsilon}=(\operatorname{Id}+\epsilon \kappa) \circ f, \quad \kappa(x, y, z)=(0,0, \beta(x, y))
$$

for some non-zero even entire function $\beta(x, y)$. The study is a bit more cumbersome when the entire perturbation has the more general form (8) with $\alpha(x)$ odd and $\beta(x, y)$ even. If $\alpha(x)$ is not odd or $\beta(x, y)$ is not even, our current technique should be restated, because ramified singularities are harder to deal with than isolated ones.

Theorem 10. Let $B_{\theta}: \mathbb{C} \rightarrow \mathbb{C}$ be the entire function

$$
B_{\theta}(r)=\int_{0}^{r} \beta(\sqrt{2 s} \cos \theta, \sqrt{2 s} \sin \theta) \mathrm{d} s
$$

Let $r(t)=z(t+h)-z(t-h)$ with $z(t)=\tanh (t / 2)$. If the function

$$
\delta_{\theta}(t)=\delta_{\theta}^{+}(t)+\delta_{\theta}^{-}(t), \quad \delta_{\theta}^{ \pm}(t)=B_{\theta \pm \omega}(r(t \pm h))
$$

has a nonremovable singularity at $t=\pi \mathrm{i}$ for some $\theta \in \mathbb{T}$, then the separatrix splits.

We note that $z(t)$ is meromorphic and its poles are the points in the set $\pi \mathrm{i}+2 \pi \mathrm{i} \mathbb{Z}$. Hence, since the function $B_{\theta}(r)$ is entire and non-zero, the compositions $\delta_{\theta}^{+}(t)$ and $\delta_{\theta}^{-}(t)$ always have a nonremovable singularity at the point $t=\pi \mathrm{i}$. Our sufficient 
condition for the splitting is that the sum $\delta_{\theta}^{+}(t)+\delta_{\theta}^{-}(t)$ be still singular at $t=\pi \mathrm{i}$, which is generic.

Proof. The function $B_{\theta}(r)$ is entire because the parity of the perturbation $\beta(x, y)$ cancels the square roots that appear in (17).

The first step is to rewrite the Melnikov function in a more convenient form. Using that $\alpha(x)=0$ and the relation $\mu(t)=-4 c s r^{\prime}(t)$, the Melnikov function (10) has the form $M(\theta, t)=-4 \operatorname{cs}\left(\Delta_{\theta}\right)^{\prime}(t)$, where

$$
\Delta_{\theta}(t)=\sum_{k \in \mathbb{Z}} \delta_{\theta}^{[k]}(t), \quad \delta_{\theta}^{[k]}(t)=B_{\theta+k \omega}(r(t+k h)) .
$$

Using that $\delta_{\theta}(t)=\delta_{\theta}^{[1]}(t)+\delta_{\theta}^{[-1]}(t)$, we shall prove that the series $\Delta_{\theta}(t)$ - and hence, the Melnikov function - has a nonremovable singularity at $t=\pi \mathrm{i}$ for some $\theta \in \mathbb{T}$.

Since the function $B_{\theta}(r)$ is entire, the composition $\delta_{\theta}^{[k]}(t)=B_{\theta+k \omega}(r(t+k h))$ is analytic but at the poles of the meromorphic function $r(t+k h)$, which are the points in the sets $\pm h-k h+\pi \mathrm{i}+2 \pi \mathrm{i} \mathbb{Z}$. Thus, the difference

$$
\Delta_{\theta}(t)-\delta_{\theta}(t)=\sum_{k \neq \pm 1} \delta_{\theta}^{[k]}(t)
$$

is analytic at $t=\pi \mathrm{i}$ for any $\theta \in \mathbb{T}$. On the other hand, by hypothesis, $\delta_{\theta}(t)$ has a nonremovable singularity at $t=\pi$ i for some $\theta \in \mathbb{T}$.

Next, we find some concrete perturbations of the form (16) that split the separatrix. For simplicity, we shall deal with perturbations such that the computation of the singular parts of the functions $\delta_{\theta}^{ \pm}(t)$ defined in (18) around their singularity $t=\pi \mathrm{i}$ can be easily analyzed. Polynomial perturbations are a natural choice. We need the following notations for the statement of the result. Given any $\beta(x, y) \in \mathbb{R}_{n}[x, y]$, we shall denote by $\sum_{l=0}^{n} \beta_{l}(x, y)$ its decomposition as a sum of homogeneous polynomials. That is, $\beta_{l}(\rho x, \rho y)=\rho^{l} \beta_{l}(x, y)$ for all $\rho \in \mathbb{R}$. Let $R_{\varphi}: \mathbb{R}^{2} \rightarrow \mathbb{R}^{2}$ be the rotation

$$
R_{\varphi}(x, y)=(x \cos \varphi-y \sin \varphi, x \sin \varphi+y \cos \varphi) .
$$

Proposition 11. If $\beta(x, y) \in \mathbb{R}_{2 n}[x, y]$ is even and $\beta_{2 n} \circ R_{2 \omega} \neq(-1)^{n} \beta_{2 n}$, then the separatrix splits under the polynomial perturbation (16).

Proof. The decomposition of the polynomial $\beta(x, y) \in \mathbb{R}_{2 n}[x, y]$ only has even terms: $\beta(x, y)=\sum_{l=0}^{n} \beta_{2 l}(x, y)$. Then the entire function $B_{\theta}: \mathbb{C} \rightarrow \mathbb{C}$ defined in (17) is the (not necessarily even) polynomial

$$
B_{\theta}(r)=\sum_{l=0}^{n} \hat{B}_{l}(\theta) r^{l+1}, \quad \hat{B}_{l}(\theta)=\frac{2^{l} \beta_{2 l}(\cos \theta, \sin \theta)}{l+1} .
$$

The point $t=\pi \mathrm{i}$ is a simple pole of the meromorphic function $z(t)=\tanh (t / 2)$, and so it becomes a pole of order $n+1$ of the functions $\delta_{\theta}^{+}(t)=B_{\theta+\omega}(z(t+2 h)-z(t))$ and $\delta_{\theta}^{-}(t)=B_{\theta-\omega}(z(t)-z(t-2 h))$. In particular, there exist some Laurent coefficients $\hat{\delta}_{1}^{ \pm}(\theta), \ldots, \hat{\delta}_{n+1}^{ \pm}(\theta)$ such that

$$
\delta_{\theta}^{ \pm}(t)=\frac{\hat{\delta}_{n+1}^{ \pm}(\theta)}{(t-\pi \mathrm{i})^{n+1}}+\cdots+\frac{\hat{\delta}_{1}^{ \pm}(\theta)}{t-\pi \mathrm{i}}+(\text { some analytic function at } t=\pi \mathrm{i}) .
$$

For instance, using that the residue of $z(t)$ at its poles is equal to 2 , we get that the dominant Laurent coefficients are $\hat{\delta}_{n+1}^{ \pm}(\theta)=(\mp 2)^{n+1} \hat{B}_{n}(\theta \pm \omega)$. 
Finally, we note that if there exists some $\theta \in \mathbb{T}$ and some index $j=1, \ldots, n+1$ such that $\hat{\delta}_{j}^{+}(\theta)+\hat{\delta}_{j}^{-}(\theta) \neq 0$, then $\delta_{\theta}(t)=\delta_{\theta}^{+}(t)+\delta_{\theta}^{-}(t)$ has a nonremovable singularity at $t=\pi \mathrm{i}$ and the separatrix splits. The functional condition $\beta_{2 n} \circ R_{2 \omega} \neq(-1)^{n} \beta_{2 n}$ is equivalent to the existence of some angle $\theta \in \mathbb{T}$ such that

$$
\beta_{2 n}(\cos (\theta+2 \omega), \sin (\theta+2 \omega)) \neq(-1)^{n} \beta_{2 n}(\cos \theta, \sin \theta)
$$

which is equivalent to the existence of $\theta$ such that $\hat{\delta}_{n+1}^{+}(\theta)+\hat{\delta}_{n+1}^{-}(\theta) \neq 0$.

Using this proposition, we shall obtain many polynomial perturbations that split the separatrix. To explain this, we introduce the complexified variables

$$
z=x+y \mathrm{i}, \quad \bar{z}=x-y \mathrm{i} .
$$

In these variables, the functional equation $\beta_{2 n} \circ R_{2 \omega}=(-1)^{n} \beta_{2 n}$ reads as $\tilde{\beta}_{2 n} \circ \tilde{R}_{2 \omega}=$ $(-1)^{n} \tilde{\beta}_{2 n}$. Here, $\tilde{R}_{\varphi}(z, \bar{z})=\left(\mathrm{e}^{\varphi \mathrm{i}} z, \mathrm{e}^{-\varphi \mathrm{i}} \bar{z}\right)$ and

$$
\tilde{\beta}_{2 n}(z, \bar{z})=\sum_{k=-n}^{n} \tilde{\beta}_{2 n}^{[k]} z^{n+k} \bar{z}^{n-k}
$$

stand for the rotation (19) and the homogeneous polynomial $\beta_{2 n}(x, y)$ in the complexified variables, respectively. The transformed polynomial $\tilde{\beta}_{2 n}(z, \bar{z})$ is still a homogeneous polynomial of degree $2 n$ because the change (20) is linear.

Lemma 12. The functional equation $\beta_{2 n} \circ R_{2 \omega}=(-1)^{n} \beta_{2 n}$ holds if and only if

$$
\tilde{\beta}_{2 n}^{[k]}\left(\mathrm{e}^{4 \omega k \mathrm{i}}-(-1)^{n}\right)=0, \quad \forall k=-n, \ldots, n .
$$

Proof. $\tilde{R}_{2 \omega}(z, \bar{z})=\left(\mathrm{e}^{2 \omega \mathrm{i}} z, \mathrm{e}^{-2 \omega \mathrm{i}} \bar{z}\right)$ maps $z^{n+k} \bar{z}^{n-k}$ onto $\mathrm{e}^{4 k \omega \mathrm{i}} z^{n+k} \bar{z}^{n-k}$.

Now we are ready to give precise statements about the splitting of the separatrix under polynomial perturbations of the form (16). For instance, we shall see that both nonresonant frequencies and high-order resonant frequencies - that is, $\omega / \pi \notin \mathbb{Q}$ or $\omega / \pi$ is an irreducible fraction with a high denominator-, are strong obstructions for the persistence of the separatrix. A homogeneous polynomial $\beta_{2 n}(x, y)$ of degree $2 n$ is rotationally invariant when it has the form

$$
\beta_{2 n}(x, y)=\tilde{\beta}_{2 n}^{[0]} z^{n} \bar{z}^{n}=\tilde{\beta}_{2 n}^{[0]}|z|^{2 n}=\tilde{\beta}_{2 n}^{[0]}\left(x^{2}+y^{2}\right)^{n}
$$

for some constant $\tilde{\beta}_{2 n}^{[0]} \in \mathbb{R}$. These polynomials are the only homogeneous ones that remain invariant under the action of the continuous group of rotations $R_{\varphi}: \mathbb{R}^{2} \rightarrow \mathbb{R}^{2}$.

THEOREM 13. If $\beta(x, y)$ is an even polynomial of degree $2 n$, then the perturbation (16) splits the separatrix in any of the following two cases:

(i) $n$ odd and $\mathrm{e}^{4 k \omega \mathrm{i}} \neq-1$ for $k=1, \ldots, n$; or

(ii) $n$ even, $\beta_{2 n}(x, y)$ not rotationally invariant, and $\mathrm{e}^{4 k \omega \mathrm{i}} \neq 1$ for $k=1, \ldots, n$.

Proof. From proposition 11 and lemma 12, we know that (21) is a necessary condition for the persistence of the separatrix. Let us check that this condition is incompatible with the two listed cases.

(i) If $n$ is odd and $\mathrm{e}^{4 k \omega \mathrm{i}} \neq-1$ for $k=1, \ldots, n$, condition (21) implies that $\tilde{\beta}_{2 n}^{[k]}=0$ for all $k=-n, \ldots, n$. Therefore, the homogeneous polynomial $\beta_{2 n}(x, y)$ is zero, which contradicts the fact that $\beta(x, y)$ has degree $2 n$.

(ii) If $n$ is even and $\mathrm{e}^{4 k \omega \mathrm{i}} \neq 1$ for $k=1, \ldots, n$, then condition (21) implies that $\tilde{\beta}_{2 n}^{[k]}=0$ for all $k \neq 0$, which contradicts the fact that $\beta(x, y)$ has degree $2 n$ and $\beta_{2 n}(x, y)$ is not rotationally invariant. 


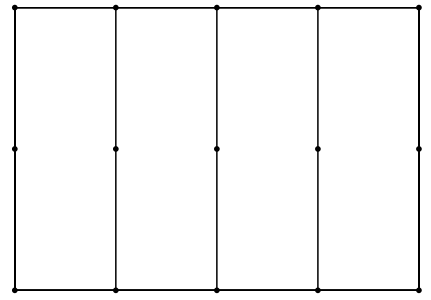

(a) $\omega=0$

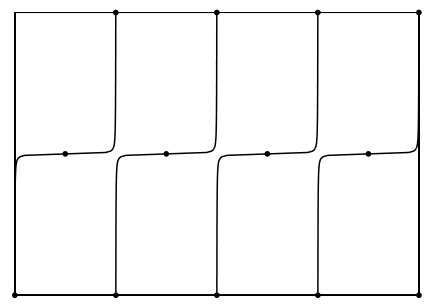

(d) $\omega=\pi / 2-10^{-3}$

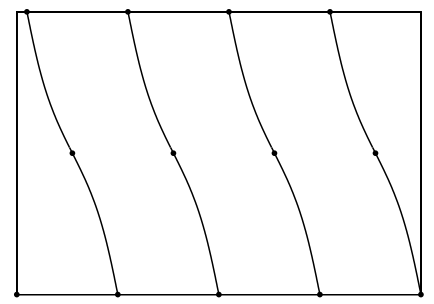

(g) $\omega=11 \pi / 20$

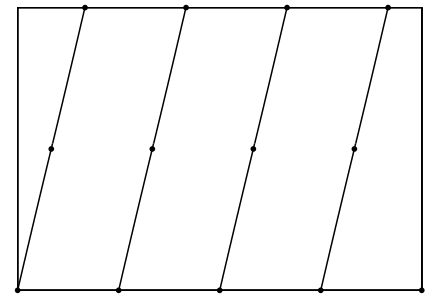

(b) $\omega=\pi / 3$

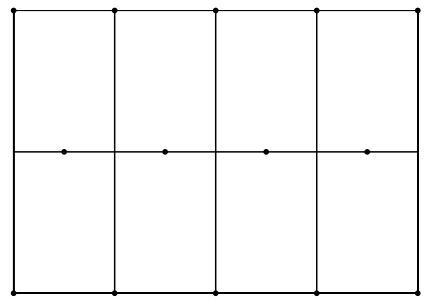

(e) $\omega=\pi / 2$

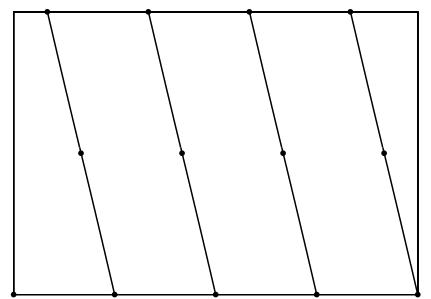

(h) $\omega=2 \pi / 3$

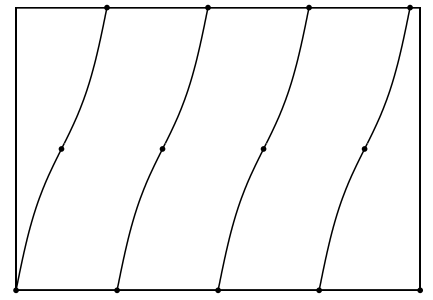

(c) $\omega=9 \pi / 20$

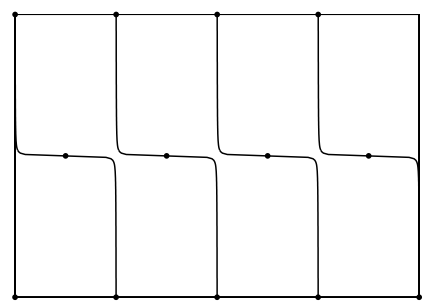

(f) $\omega=\pi / 2+10^{-3}$

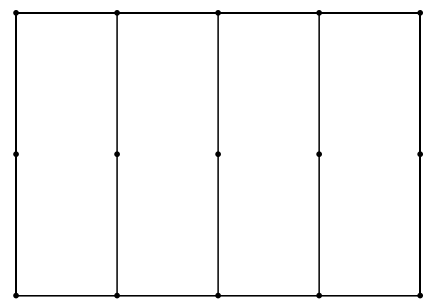

(i) $\omega=\pi$

FIG. 4. The only bifurcation of $\tilde{Z}$ in the range $0 \leq \omega \leq \pi$ under the perturbation $\kappa(x, y, z)=$ $(0, x, 0)$ takes place at the singular frequency $\omega=\pi / \overline{2}$. These pictures show this bifurcation for $h=1$. The symmetric points move as the frequency $\omega$ varies.

It would be interesting to know whether there exist some entire perturbations of the form (16) that preserve the separatrix. Of course, such perturbations can not verify the sufficient condition for splitting given in theorem 10 . We have not found any perturbation of this kind, which is not so strange because in similar contexts, related with other McMillan maps, they simply do not exist. An area-preserving example of this situation can be found in [13], and a high-dimensional symplectic one in [14].

7. Bifurcations of the zero-level set in an example. In this section, we shall study the bifurcations in $\omega \in \mathbb{T}$ of the topological shape of the zero-set $Z \subset \mathbb{T} \times \mathbb{R}$ for the perturbation $\kappa(x, y, z)=(0, x, 0)$. We note that, according to lemma 5 and remark $4, Z$ contains (and is symmetric with regard to) the eight symmetric points shown in figure 3 and it is also $(\pi / 2,0)$-periodic.

Based on detailed numerical computations and several analytical arguments, we conjecture that 0 is a singular value of the Melnikov function if and only if $\omega= \pm \pi / 2$, and so the only bifurcations of $Z=M^{-1}(0)$ take place at those values. For instance, we show in figure 4 the numerically computed shape of $\tilde{Z}$ for several values of the frequency in the range $0 \leq \omega \leq \pi$.

We give a dynamical interpretation of these Melnikov-like results. If $\omega \neq \pm \pi / 2$, the set of primary intersections $P_{\epsilon}=Z+\mathrm{O}(\epsilon)$ consists in four vertical curves for $\epsilon$ 
small enough, see theorem 1 . Each curve has a symmetric twin, because $P_{\epsilon}$ is invariant under the axial symmetry $S(x, y, z)=(-x,-y, z)$. The fixed sets of the reversors are

$$
\begin{aligned}
\text { Fix } R_{\epsilon} & =\left\{(x, y, z) \in \mathbb{R}^{3}: y=\epsilon x / 2, z=0\right\}, \\
\operatorname{Fix} T_{\epsilon} & =\left\{(x, y, z) \in \mathbb{R}^{3}: x=0, z=0\right\} .
\end{aligned}
$$

Hence, two heteroclinic vertical curves cross a curve $\mathrm{O}(\epsilon)$-close to the $x$-axis and the other pair of heteroclinic curves cross the $y$-axis. The rotation number of these vertical curves is equal to $\omega$ in the range $-\pi / 2<\omega<\pi / 2$, but it jumps to $\omega \mp \pi$ when the bifurcation values $\omega= \pm \pi / 2$ are crossed. The shape of the primary set when $\omega= \pm \pi / 2$ it is not completely clear, because then 0 is a singular value of the Melnikov function and theorem 1 can not be applied. This is an open question.

The rest of the section is devoted to present some rigorous results supporting the previous conjecture, although we have found a complete proof only for $h \geq h_{0} \approx 2.28$. Nevertheless, we have been able to prove the following results. If $\omega$ is a regular frequency (that is, if 0 is a regular value of the Melnikov function), then $Z$ contains just four vertical curves. Otherwise, we say that $\omega$ is a singular frequency and $Z$ contains the four vertical curves jointly with the images and pre-images of exactly one horizontal straight line, in which case the degenerate zeros of the Melnikov function are just the points in the intersections between the horizontal and vertical curves. The number of singular frequencies is finite. The frequencies $\omega=0$ and $\omega=\pi$ are regular, whereas $\omega= \pm \pi / 2$ are singular and, in addition, they are the only singular ones when the characteristic exponent is big enough: $h \geq h_{0}:=\log 16-\log (\sqrt{113}-9) \approx 2.28$.

In order to lighten the computations, we write the Melnikov function in its simplest form. Let $\chi: \mathbb{R} \rightarrow \mathbb{R}$ be the function

$$
\chi(t)=\frac{4 c^{2} s^{2}}{\cosh ((t+h) / 2) \cosh ^{2}(t / 2) \cosh ((t-h) / 2)} .
$$

Then, using that $\alpha(x)=x$ and $\beta(x, y)=0$, the Melnikov function (10) becomes

$$
M(\theta, t)=a(t) \sin 2 \theta+b(t) \cos 2 \theta
$$

where $a(t)$ and $b(t)$ are given by the absolutely convergent series

$$
a(t)=\sum_{k \in \mathbb{Z}} \cos (2 k \omega) \chi(t+k h), \quad b(t)=\sum_{k \in \mathbb{Z}} \sin (2 k \omega) \chi(t+k h) .
$$

We also introduce the complex-valued function

$$
E(t)=E_{\omega}(t)=\sum_{k \in \mathbb{Z}} \mathrm{e}^{2 k \omega \mathrm{i}} \chi(t+k h)=a(t)+b(t) \mathrm{i}
$$

which plays a crucial rôle in the digression because of the relation

$$
\partial_{\theta} M(\theta, t) / 2+M(\theta, t) \mathrm{i}=E(t) \mathrm{e}^{2 \theta \mathrm{i}} .
$$

This relation has interesting consequences. For instance, if $\left(\theta_{0}, t_{0}\right) \in \mathbb{T} \times \mathbb{R}$ is a degenerate zero of the Melnikov function, $E\left(t_{0}\right)$ must be zero. In particular, 0 is a regular value of the Melnikov function when $E(t)$ has no real zeros. Therefore, we are naturally led to the study of the sets

$$
\Omega=\Omega_{h}=\left\{\omega \in \mathbb{T}: E_{\omega}(t) \text { has some real zero }\right\}, \quad h>0 .
$$


Their main properties are addressed in the following lemma, whose proof is deferred to appendix A. The proof is based on some nice properties of quasielliptic functions that can be deduced from elementary facts of complex variable theory contained in any basic textbook like, for instance, [33].

LEMMA 14. Given any $h>0$, the set (26) is finite, $\pi$-periodic, and symmetric: $\Omega=-\Omega$. If the function (24) has some real zero, all of them are simple and the set of its real zeros is either $h \mathbb{Z}$ or $h / 2+h \mathbb{Z}$, so $\Omega$ is the disjoint union of the sets

$$
\Omega^{0}=\left\{\omega \in \mathbb{T}: E_{\omega}(0)=0\right\}, \quad \Omega^{1}=\left\{\omega \in \mathbb{T}: E_{\omega}(h / 2)=0\right\} .
$$

Besides, $\pm \pi / 2 \in \Omega^{1}, 0 \notin \Omega$, and $\pi \notin \Omega$. Finally, $\Omega^{1}=\{ \pm \pi / 2\}$ for $h \geq h_{1}:=$ $2 \log \frac{20}{9} \approx 1.60$ and $\Omega^{0}=\emptyset$ for $h \geq h_{0}:=\log 16-\log (\sqrt{113}-9) \approx 2.28$.

Conjecture 15. $\Omega=\Omega^{1}=\{ \pm \pi / 2\}$ and $\Omega^{0}=\emptyset$ for all $h>0$.

We present in appendix B a strong numerical evidence on this conjecture.

Lemma 16. Let $E: \mathbb{R} \rightarrow \mathbb{C}$ be an analytic function such that $E(-t)=\overline{E(t)}$.

(i) If $E(t)$ has no real zeros, then there exists a unique odd analytic function $\varphi: \mathbb{R} \rightarrow \mathbb{R}$ and an integer $n \in\{0,1\}$ such that $E(t)=|E(t)| \mathrm{e}^{(\varphi(t)+\pi n) \mathrm{i}}$ for all $t \in \mathbb{R}$.

(ii) If $E(t)$ has no real multiple zeros, then there exists a unique odd analytic function $\varphi: \mathbb{R} \rightarrow \mathbb{R}$ and a function $n: \mathbb{R} \rightarrow\{0,1\}$ such that $E(t)=|E(t)| \mathrm{e}^{(\varphi(t)+\pi n(t)) \mathrm{i}}$ for all $t \in \mathbb{R}$. The function $n(t)$ is constant, but at the zeros of $E(t)$.

Proof. (i) If a function is analytic and never zero on a convex subset of the complex plane, then it has an analytic argument on that convex subset. This is an elementary result in complex variable theory, see $[4, \S 2.1]$. Let $\varphi(t)$ be an analytic argument of $E(t) / E(0)$; that is, any analytic function $\varphi: \mathbb{R} \rightarrow \mathbb{R}$ such that

$$
E(t)=E(0)|E(t) / E(0)| \mathrm{e}^{\varphi(t) \mathrm{i}}=|E(t)| \mathrm{e}^{(\varphi(t)+\pi n) \mathrm{i}} .
$$

Obviously, $n=0$ if $E(0)>0$ and $n=1$ if $E(0)<0$. The argument is not unique, but it is determined up to a multiple of $2 \pi$; that is, it is determined once we choose the value of $\varphi(0)$ from the set $2 \pi \mathbb{Z}$. The condition $E(-t)=\overline{E(t)}$ implies that $\varphi(-t)+\varphi(t)=$ $2 \varphi(0)$ for all real $t$. If we want an odd argument, $\varphi(0)=0$ in the only possible choice.

(ii) It suffices to realize that, at any simple zero, the argument undergoes a jump by a multiple of $\pi$. When these jumps are stored in the discrete-valued function $n(t)$, the function $\varphi(t)$ remains analytic.

These two lemmas are the basis for the next theorem, in which the shape and bifurcations of the zero-level set $Z=M^{-1}(0) \subset \mathbb{T} \times \mathbb{R}$ are described.

THEOREM 17. Let $Z$ be the zero-level set of the Melnikov function (23). Let $\Omega=\Omega^{0} \cup \Omega^{1}$ be the decomposition of the set (26) given in lemma 14. Let $\omega \in \mathbb{T}$. There exist a unique odd analytic function $\bar{\theta}_{\omega}: \mathbb{R} \rightarrow \mathbb{R}$ such that

(i) If $\omega \notin \Omega$, then $Z=\left\{\theta=\bar{\theta}_{\omega}(t) \quad(\bmod \pi / 2)\right\}$ and $\omega$ is a regular frequency.

(ii) If $\omega \in \Omega^{j}$, then $Z=\{t=j h / 2(\bmod h)\} \cup\left\{\theta=\bar{\theta}_{\omega}(t) \quad(\bmod \pi / 2)\right\}$ and $\omega$ is a singular frequency. The degenerate zeros of the Melnikov function are just the points in the intersections between the horizontal lines and the vertical curves.

(iii) The function $\bar{\Theta}(t, \omega):=\bar{\theta}_{\omega}(t)$ is analytic on $\mathbb{R} \times(\mathbb{T} \backslash \Omega)$.

(iv) If $\omega=0 \quad(\bmod \pi / 2)$, then $\bar{\theta}_{\omega}(t) \equiv 0$.

(v) $\bar{\theta}_{\omega}(t+h)=\bar{\theta}_{\omega}(t)+\omega \quad(\bmod \pi / 2)$.

(vi) $\bar{\theta}_{\omega}(h / 2-t)+\bar{\theta}_{\omega}(h / 2+t)=\omega \quad(\bmod \pi / 2)$.

(vii) $\bar{\theta}_{-\omega}(t)=-\bar{\theta}_{\omega}(t)$ and $\bar{\theta}_{\omega+\pi}(t)=\bar{\theta}_{\omega}(t)$.

Proof. Sometimes, we do not write explicitly the dependence on the frequency. Using that $a(t)$ is even and $b(t)$ is odd, we see that the function (24) verifies the relation $E(-t)=\overline{E(t)}$. This is important, because it was a hypothesis in lemma 16 
(i) If $\omega \notin \Omega$, then $E(t)$ has no real zeros, so $\omega$ is a regular frequency. It remains to prove that $Z$ is composed by four vertical curves of the form $\{\theta=\bar{\theta}(t) \quad(\bmod \pi / 2)\}$ for some function $\bar{\theta}(t)$. Let $\bar{\theta}: \mathbb{R} \rightarrow \mathbb{R}$ be the odd analytic function $\bar{\theta}(t)=-\varphi(t) / 2$, where $\varphi(t)=\arg (E(t) / E(0))$ is the argument introduced in lemma 16. Let $n$ be the integer mentioned in the same lemma. Using that $E(t)$ has no real zeros jointly with relation (25), we have

$$
\begin{aligned}
Z=M^{-1}(0) & =\left\{(\theta, t) \in \mathbb{T} \times \mathbb{R}: E(t) \mathrm{e}^{2 \theta \mathrm{i}} \in \mathbb{R}\right\} \\
& =\{(\theta, t) \in \mathbb{T} \times \mathbb{R}: \sin (\varphi(t)+\pi n+2 \theta)=0\} \\
& =\{(\theta, t) \in \mathbb{T} \times \mathbb{R}: \theta=\bar{\theta}(t) \quad(\bmod \pi / 2)\} .
\end{aligned}
$$

(ii) We know that $E(t)$ has no real multiple zeros. Let $\varphi(t)$ and $n(t)$ be the functions given in lemma 16. Let $\bar{\theta}(t)=-\varphi(t) / 2$. Then

$$
\begin{aligned}
Z & =\left\{(\theta, t) \in \mathbb{T} \times \mathbb{R}: E(t) \mathrm{e}^{2 \theta \mathrm{i}} \in \mathbb{R}\right\} \\
& =\{(\theta, t): E(t)=0\} \cup\{(\theta, t): \sin (\varphi(t)+\pi n(t)+2 \theta)=0\} \\
& =\{t=j h / 2 \quad(\bmod h)\} \cup\{\theta=\bar{\theta}(t) \quad(\bmod \pi / 2)\} .
\end{aligned}
$$

Therefore, $Z=M^{-1}(0)$ contains four vertical curves that intersect infinitely many horizontal straight lines. Obviously, these intersections are degenerate zeros of the Melnikov function. Next, we shall prove that the other zeros are nondegenerate.

Let $\left(\theta_{0}, t_{0}\right)$ be a zero of the Melnikov function not contained in any horizontal line: $M\left(\theta_{0}, t_{0}\right)=0$ and $E\left(t_{0}\right) \neq 0$. Then $\partial_{\theta} M\left(\theta_{0}, t_{0}\right)=2 E\left(t_{0}\right) \mathrm{e}^{2 \theta_{0} \mathrm{i}} \neq 0$, see $(25)$.

On the other hand, let $\left(\theta, t_{1}\right)$ a point contained in some horizontal line: $M\left(\theta, t_{1}\right)=$ $0, E\left(t_{1}\right)=0$ and $E^{\prime}\left(t_{1}\right) \neq 0$. Again from relation $(25)$, we get $\partial_{\theta} M\left(\theta, t_{1}\right)=0$ and $\partial_{t} M\left(\theta, t_{1}\right)=\Im E^{\prime}\left(t_{1}\right) \mathrm{e}^{2 \theta \mathrm{i}}$, where $\Im$ denotes imaginary part. Hence, the degenerate zeros on the horizontal line $\left\{t=t_{1}\right\}$ are just the ones that verify the condition $\Im E^{\prime}\left(t_{1}\right) \mathrm{e}^{2 \theta \mathrm{i}}=0$. But, since $E^{\prime}\left(t_{1}\right) \neq 0$, there are exactly four of such angles $\theta \in \mathbb{T}$. These four angles are the ones corresponding to the four intersections of the horizontal line $\left\{t=t_{1}\right\}$ with the four vertical curves $\{\theta=\bar{\theta}(t) \quad(\bmod \pi / 2)\}$.

(iii) Level sets associated to regular values of analytic functions vary in an analytic way under analytic perturbations.

(iv) If $\omega=0 \quad(\bmod \pi / 2)$, then $\sin (2 k \omega)=0$ for all $k$, and $b(t)=\Im E(t) \equiv 0$.

(v) This has to do with the fact that the zero-level set $Z$ is $(\omega, h)$-periodic. Given any $t \in \mathbb{R}$, we consider the slice $Z_{t}=\{\theta \in \mathbb{T}:(\theta, t) \in Z\}$. If $E(t) \neq 0$, then

$$
\begin{aligned}
Z_{t+h} & =\{\theta \in \mathbb{T}: \theta=\bar{\theta}(t+h) \quad(\bmod \pi / 2)\}, \\
Z_{t}+\omega & =\{\theta \in \mathbb{T}: \theta=\bar{\theta}(t)+\omega \quad(\bmod \pi / 2)\} .
\end{aligned}
$$

But these two sets coincide, due to the $(\omega, h)$-periodicity of $Z$, and so we obtain that $\bar{\theta}(t+h)=\bar{\theta}(t)+\omega \quad(\bmod \pi / 2)$ for any real $t$ such that $E(t) \neq 0$. Indeed, by analytic continuation, this equality holds for any real $t$.

(vi) It follows directly from the previous item and the odd character of $\bar{\theta}(t)$ : $\bar{\theta}(h / 2-t)+\bar{\theta}(h / 2+t)=-\bar{\theta}(t-h / 2)+\bar{\theta}(t-h / 2)+\omega=\omega \quad(\bmod \pi / 2)$.

(vii) Firstly, $\bar{\theta}_{-\omega}(t)=-\frac{1}{2} \arg E_{-\omega}(t)=-\frac{1}{2} \arg \overline{E_{\omega}(t)}=\frac{1}{2} \arg E_{\omega}(t)=-\bar{\theta}_{\omega}(t)$. Secondly, $\bar{\theta}_{\omega+\pi}(t)=-\frac{1}{2} \arg E_{\omega+\pi}(t)=-\frac{1}{2} \arg E_{\omega}(t)=\bar{\theta}_{\omega}(t)$.

Remark 9. The numerical computations show that $\bar{\theta}_{\omega}(t+h)=\bar{\theta}_{\omega}(t)+\omega$ only holds in the range $-\pi / 2<\omega<\pi / 2$, see figure 4 . This does not contradict item (v) in theorem 17. 
Remark 10. Similar results hold for the perturbation $\kappa(x, y, z)=\left(0,0, y^{2}\right)$. In that case, it turns out that the Melnikov function has the form

$$
M(\theta, t)=\hat{a}(t) \sin 2 \theta+\hat{b}(t) \cos 2 \theta+\hat{c}(t)
$$

for some absolutely convergent series $\hat{a}(t), \hat{b}(t)$, and $\hat{c}(t)$. The analytical study is harder because of the additional third term - compare with (23). We have numerically checked that the only bifurcations take place at the singular frequencies $\omega \in\{0, \pm \pi / 2, \pi\}$, whereas the zero-level set still contains just four vertical curves for regular frequencies.

8. Conclusion and open problems. In this paper, we have obtained several analytical results about the splitting of separatrices under perturbations of some integrable volume-preserving maps using a discrete version of the Melnikov method. The integrable maps have a bi-dimensional heteroclinic connection of spherical shape between two fixed points of saddle-focus type. We have bounded the topological complexity of the primary heteroclinic set under some polynomial perturbations. We have also given a sufficient condition for the splitting of the separatrices under some entire perturbations. Finally, we have obtained a complete picture of the bifurcations that take place under a simple perturbation. In spite of these results, many unsolved questions remain. We indicate three.

We conjecture that the separatrix splitting studied in this paper is exponentially small in the characteristic exponent, but the rôle of the frequency is still unclear. Several examples of the effect that resonant frequencies can have in the dynamics of 3D maps near Hopf-saddle-node bifurcations can be found in [9, 10], although not related to a problem about the splitting of separatrices. One could guest an asymptotic exponentially small formula for the splitting using a multiple-precision arithmetic, like in [30]. Such formulae for the splitting of one-dimensional heteroclinic connections between saddle-focus fixed points of volume-preserving systems have already been found in [2] (for maps) and [7] (for flows), but we do not know any similar formula for the bi-dimensional case.

Another question is: what about Šil'nikov-like bifurcations in the discrete setting? The perturbation of a spheromak structure in 3D flows is a classical setup to study Šil'nikov bifurcations. Some results about the volume-preserving case are contained in [11]. It is natural to consider the discrete version of this problem, although the problem seems qualitatively more complicated.

Finally, it would be interesting to study some questions about transport. As a first step, we should compute the geometric flux through the perturbed separatrices. The $\mathrm{O}(\epsilon)$-term of this flux can be computed by integrating certain Melnikov two-form over a suitable region, see [23]. Next, we could follow the ideas introduced in [25], although we must take into account that the scenario for maps is richer than the one for flows. For instance, we recall that equatorial and bubble-type heteroclinic curves can not appear in autonomous flows.

Acknowledgments. This work was completed while HL was a visitor at the University of Texas at Austin, whose hospitality is gratefully acknowledged. Useful conversations with Jaume Amorós, Amadeu Delshams, Rafael de la Llave, James Meiss, Carles Simó, and Jordi Villanueva are also acknowledged.

\section{Appendix A. Proof of lemma 14.}

Here, we study the existence of real zeros of the function (24). This function has many properties similar to the ones of elliptic functions and so we shall study its zeros 
using tools typical in the theory of elliptic functions.

We list in the next lemma some basic properties of the function $E(t)$, including that $E(t)$ is meromorphic, $2 \pi \mathrm{i}$-periodic and $h$-quasiperiodic, and so quasielliptic.

LEMma 18. The function $E(t)=E_{\omega}(t)=\sum_{k \in \mathbb{Z}} \mathrm{e}^{2 k \omega \mathrm{i}} \chi(t+k h)$ verifies that:

(i) It is meromorphic in the complex plane and its poles are the points in the set $\mathcal{P}=\pi \mathrm{i}+h \mathbb{Z}+2 \pi \mathrm{i} \mathbb{Z}$ (double ones).

(ii) $E(t+2 \pi \mathrm{i})=E(t)$ and $E(t+h)=\mathrm{e}^{-2 \omega \mathrm{i}} E(t)$ for all complex $t$.

(iii) $E(-\bar{t})=\overline{E(t)}$ for all complex $t$.

(iv) If $\omega=\pi / 2(\bmod \pi)$, the points $t=h / 2(\bmod h)$ are the only real zeros of $E(t)$, all of them being simple ones.

(v) If $\omega=0(\bmod \pi)$, then $E(t)$ has no real zeros.

(vi) $E_{-\omega}(t)=\overline{E_{\omega}(\bar{t})}$ and $E_{\omega+\pi}(t)=E_{\omega}(t)$.

Proof. (i) The function $\chi(t)$ is meromorphic in the complex plane and its poles are the points in $\pi \mathrm{i}+2 \pi \mathrm{i} \mathbb{Z}$ (double ones) and $\pi \mathrm{i} \pm h+2 \pi \mathrm{i} \mathbb{Z}$ (simple ones).

(ii) The function $E(t)$ is $2 \pi \mathrm{i}$-periodic because so is $\chi(t)$. On the other hand,

$$
E(t+h)=\sum_{k \in \mathbb{Z}} \mathrm{e}^{2 k \omega \mathrm{i}} \chi(t+k h+h)=\sum_{k \in \mathbb{Z}} \mathrm{e}^{2(k-1) \omega \mathrm{i}} \chi(t+k h)=\mathrm{e}^{-2 \omega \mathrm{i}} E(t) .
$$

(iii) We recall that $E(t)=a(t)+b(t) \mathrm{i}$, where $a(t)$ and $b(t)$ are real analytic functions such that $a(t)$ is even and $b(t)$ is odd. Hence, $E(-\bar{t})=a(-\bar{t})+b(-\bar{t}) \mathrm{i}=$ $a(\bar{t})-b(\bar{t}) \mathrm{i}=\overline{a(t)}-\overline{b(t)} \mathrm{i}=\overline{E(t)}$.

(iv) In this case, $\mathrm{e}^{2 k \omega \mathrm{i}}=-1$, and so $E(t+h)=-E(t)$. Thus, $E(t)$ becomes an elliptic function with periods $2 h$ and $2 \pi$ i. Besides, $E(t)$ has order four, because it has just four poles (counted with multiplicity) on any cell with periods $2 h$ and $2 \pi \mathrm{i}$, see (i).

Using that the elliptic function

$$
E(t)=\sum_{k \in \mathbb{Z}}(-1)^{k} \chi(t+k h)
$$

is even, $h$-antiperiodic and $2 \pi \mathrm{i}$-periodic, we get that $E(t)$ vanishes at the four points $h / 2,3 h / 2, h / 2+\pi \mathrm{i}$, and $3 h / 2+\pi \mathrm{i}$. For instance, $E(h / 2)=E(-h / 2)=-E(h / 2)$, so $E(h / 2)=0$. But we know that $E(t)$ has exactly four zeros (counted with multiplicity) on each cell with periods $2 h$ and $2 \pi$ i. Since the previous four zeros belong to the same cell, they are the only ones (modulo periodicities), and they are simple.

(v) $\chi(t)>0$ in $\mathbb{R}$. Thus, if $\mathrm{e}^{2 \omega \mathrm{i}}=1, E(t)=\sum_{k \in \mathbb{Z}} \chi(t+k h)>0$ for any real $t$.

(vi) Firstly, $E_{-\omega}(t)=\sum_{k \in \mathbb{Z}} \overline{\mathrm{e}^{2 k \omega \mathrm{i}} \overline{\chi(t+k h)}}=\overline{\sum_{k \in \mathbb{Z}} \mathrm{e}^{2 k \omega \mathrm{i}} \chi(\bar{t}+k h)}=\overline{E_{\omega}(\bar{t})}$, because $\chi(t)$ is real analytic. The second property is trivial.

Next, we gain some insight on the structure of the complex zeros of the quasielliptic function $E(t)$. Roughly speaking, we state in the following lemma that $E(t)$ has order two and its zeros look like in figure 5. The proof is adapted from similar proofs about elliptic functions.

LEMma 19. The quasielliptic function $E(t)$ has order two; that is, it has two zeros in any cell with periods $h$ and $2 \pi \mathrm{i}$. Let $t_{1}$ and $t_{2}$ be the zeros in any cell. Then

$$
t_{1}+t_{2} \in 2 \omega \mathrm{i}+h \mathbb{Z}+2 \pi \mathrm{i} \mathbb{Z}
$$

Besides, the set

$$
\mathcal{T}=\left\{t_{1}, t_{2}\right\}+h \mathbb{Z}+2 \pi \mathrm{i} \mathbb{Z}
$$




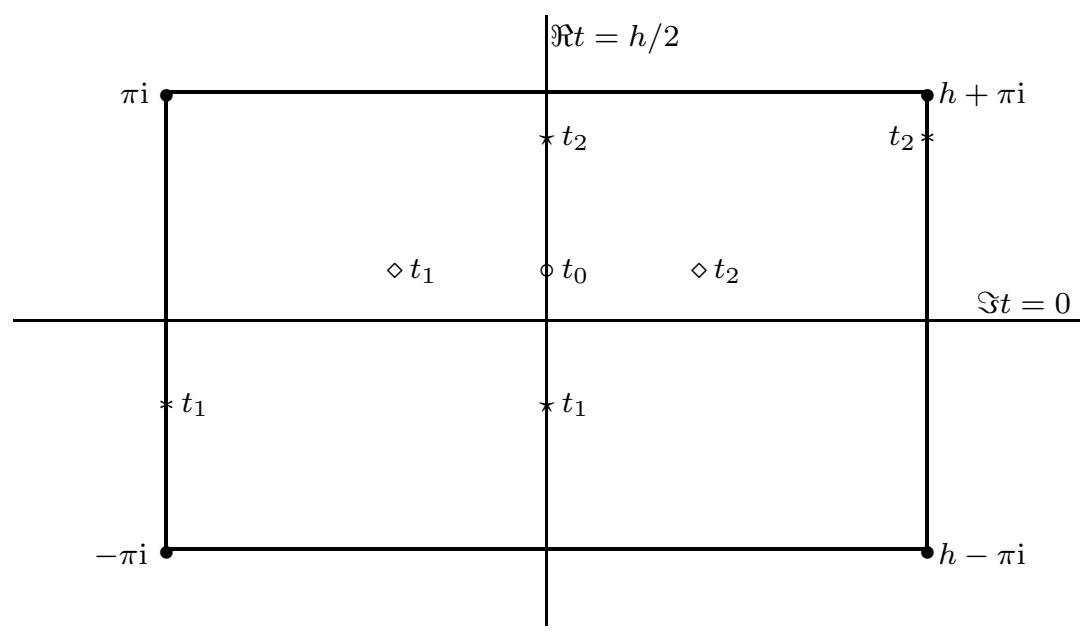

FiG. 5. The three scenarios for the set of zeros of the quasielliptic function E(t). First $(\star)$ : The zeros are on the lines $\{\Re t=h / 2(\bmod h)\}$. Second $(\diamond):$ The zeros have the same imaginary part modulo $2 \pi \mathrm{i}$. Third $(*)$ : The zeros are on the lines $\{\Re t=0(\bmod h)\}$. The double poles are marked with the symbol $\bullet$. The middle point is $t_{0}=h / 2+\omega \mathrm{i}$ in the three cases, because $t_{1}+t_{2} \in 2 \omega \mathrm{i}+h \mathbb{Z}+2 \pi \mathrm{i} \mathbb{Z}$.

formed by the complex zeros of $E(t)$ is symmetric with regard to the vertical lines $\{\Re t=0 \quad(\bmod h)\}$ and $\{\Re t=h / 2 \quad(\bmod h)\}$.

Proof. We recall the following version of the Argument Principle [33, §6.3]. Let $C$ be a contour in the complex plane, let $f(t)$ be a function analytic inside and on $C$, let $g(t)$ be a meromorphic function without zeros or poles on $C$, and let $t_{1}, \ldots, t_{J}$ and $p_{1}, \ldots, p_{K}$ be the zeros and poles of $g(t)$ in the interior of $C$, repeated as many times as their multiplicities and orders, respectively. Then

$$
\frac{1}{2 \pi \mathrm{i}} \oint_{C} f(t) \frac{g^{\prime}(t)}{g(t)} \mathrm{d} t=\sum_{j} f\left(t_{j}\right)-\sum_{k} f\left(p_{k}\right) .
$$

This version of the principle assumes that the contour has no self-intersections, and that it is oriented counter-clockwise.

If we choose any cell of periods $h$ and $2 \pi \mathrm{i}$ as the contour $C$, and put $f(t)=1$, $g(t)=E(t)$ in (28), we get that $E(t)$ has $J=K=2$ zeros in the cell, because the integrals of the quotient $E^{\prime}(t) / E(t)$ over opposites sides of the cell cancel out. (We recall that $E(t)$ has exactly one double pole on each cell.)

Let $t_{1}$ and $t_{2}$ be the zeros and $p$ be the double pole of $E(t)$ in a cell $C$. Let the corners of the cell be $s, s+h, s+h+2 \pi \mathrm{i}, s+2 \pi \mathrm{i}$. Now, if we keep the same contour, but take $f(t)=t$ and $g(t)=E(t)$, we get that

$$
\begin{aligned}
t_{1}+t_{2}-2 p & =\frac{1}{2 \pi \mathrm{i}}\left(\int_{s}^{s+h}+\int_{s+h}^{s+h+2 \pi \mathrm{i}}+\int_{s+h+2 \pi \mathrm{i}}^{s+2 \pi \mathrm{i}}+\int_{s+2 \pi \mathrm{i}}^{s}\right) \frac{t E^{\prime}(t)}{E(t)} \mathrm{d} t \\
& =\frac{1}{2 \pi \mathrm{i}}\left(h \int_{s}^{s+2 \pi \mathrm{i}} \frac{E^{\prime}(t)}{E(t)} \mathrm{d} t-2 \pi \mathrm{i} \int_{s}^{s+h} \frac{E^{\prime}(t)}{E(t)} \mathrm{d} t\right) \\
& =\left.\frac{h}{2 \pi \mathrm{i}} \log E(t)\right|_{s} ^{s+2 \pi \mathrm{i}}+\left.\log E(t)\right|_{s+h} ^{s}
\end{aligned}
$$

on making use of the quasiperiodic properties of $E(t)$. 
Using again the quasiperiodicities $E(s+2 \pi \mathrm{i})=E(s)$ and $E(s+h)=\mathrm{e}^{-2 \omega \mathrm{i}} E(s)$, we see that $\log E(t)]_{s}^{s+2 \pi \mathrm{i}} \in 2 \pi \mathrm{i} \mathbb{Z}$ and $\left.\log E(t)\right]_{s+h}^{s} \in 2 \omega \mathrm{i}+2 \pi \mathrm{i} \mathbb{Z}$. Therefore,

$$
t_{1}+t_{2} \in 2 \omega \mathrm{i}+2 p+h \mathbb{Z}+2 \pi \mathrm{i} \mathbb{Z} .
$$

Now, relation (27) follows because we know that the double pole $p \in \pi \mathrm{i}+h \mathbb{Z}+2 \pi \mathrm{i} \mathbb{Z}$.

Finally, the specular symmetries with regard to the vertical lines are a direct consequence of the relations $E(-\bar{t})=\overline{E(t)}$ and $E(t+h)=\mathrm{e}^{-2 \omega \mathrm{i}} E(t)$.

Using this lemma, we realize that there are just three possible scenarios for the set of complex zeros of the quasielliptic function $E(t)$, which are listed in the caption of figure 5 . We have numerically checked that only the first scenario takes place, but we have not found a proof. Therefore, in the following lemma we can only deduce that the set of real zeros of $E(t)$ is either $h \mathbb{Z}$ or $h / 2+h \mathbb{Z}$, although we suspect that the case $h \mathbb{Z}$ is a mirage.

LEMMA 20. If $E(t)$ has some real zero, all of them are simple and the set of its real zeros is either $h \mathbb{Z}$ or $h / 2+h \mathbb{Z}$.

Proof. We note that $E(t)$ can not have neither a double real zero nor two different real zeros modulo $h$. On the contrary, we could take $t_{1}, t_{2} \in \mathbb{R}$ in lemma 19 , so that $\mathbb{R} \ni t_{1}+t_{2} \in 2 \omega \mathrm{i}+h \mathbb{Z}+2 \pi \mathrm{i} \mathbb{Z}$. But this would imply that $\omega=0 \quad(\bmod \pi)$, and then $E(t)$ has no real zeros, see lemma 18 . Therefore, the set of real zeros has the form $t_{*}+h \mathbb{Z}$ for some single real zero $t_{*} \in[0, h)$. But, since $t_{*}+h \mathbb{Z}$ must be symmetric with regard to the points 0 and $h / 2$, there are only two possibilities: either $t_{*}=0$ or $t_{*}=h / 2$.

As a by-product of this lemma, the set defined in (26) can also be defined as

$$
\Omega=\Omega_{h}=\left\{\omega \in \mathbb{T}: E_{\omega}(0) E_{\omega}(h / 2)=0\right\} .
$$

To prove lemma 14, it suffices to check that: 1) $\Omega$ is finite; 2) $E_{\omega}(0) \neq 0$ for all $h \geq h_{0}$ and all $\omega \in \mathbb{T}$; and 3$) E_{\omega}(h / 2) \neq 0$ for all $h \geq h_{1}$ and all $\omega \neq \pm \pi / 2$.

Lemma 21. The set $\Omega=\left\{\omega \in \mathbb{T}: E_{\omega}(0) E_{\omega}(h / 2)=0\right\}$ is finite.

Proof. It follows from the fact that, once fixed any $h>0$, the function

$$
\mathbb{T} \ni \omega \mapsto E_{\omega}(0) E_{\omega}(h / 2) \in \mathbb{C}
$$

is analytic, but not identically zero since the series $E_{0}(t)=\sum_{k \in \mathbb{Z}} \chi(t+k h)$ is positive for all real $t$.

LEMMA 22. If $h \geq h_{0}:=\log 16-\log (\sqrt{113}-9)$, then $E_{\omega}(0)>0$ for all $\omega \in \mathbb{T}$.

Proof. We introduce the positive quantities

$$
\chi_{k}=\chi_{k}(h):=\chi(k h)=\frac{4 \cosh ^{2}(h / 2) \sinh ^{2}(h / 2)}{\cosh ((k+1) h / 2) \cosh ^{2}(k h / 2) \cosh ((k-1) h / 2)}
$$

where $\chi(t)$ is the function given in (22). Our goal is to prove that

$$
E_{\omega}(0)=\sum_{k \in \mathbb{Z}} \mathrm{e}^{2 k \omega \mathrm{i}} \chi_{k}=\chi_{0}+2 \sum_{k \geq 1} \cos (2 k \omega) \chi_{k}>0
$$

for all $h \geq h_{0}$ and $\omega \in \mathbb{T}$. Here, we have used the symmetry $\chi_{-k}=\chi_{k}$. Since $\max _{\omega \in \mathbb{T}}|\cos (2 k \omega)|=1$, in order to prove the lemma it suffices to establish that

$$
2 \sum_{k \geq 1} \chi_{k}(h)<\chi_{0}(h), \quad \forall h \geq h_{0}:=\log 16-\log (\sqrt{113}-9) .
$$


The rest of the proof is devoted to obtain this bound. If we work with the multiplicative variable $x=\mathrm{e}^{-h} \in(0,1)$, then $\chi_{0}=\chi_{0}(x)=(1-x)^{2} / x$ and

$$
\chi_{k}=\chi_{k}(x)=\frac{4\left(1-x^{2}\right)^{2} x^{2 k-2}}{\left(1+x^{k+1}\right)\left(1+x^{k}\right)^{2}\left(1+x^{k-1}\right)}<4\left(1-x^{2}\right)^{2} x^{2 k-2}
$$

for any $k \geq 1$ and $x \in(0,1)$. In particular,

$$
\sum_{k \geq 1} \chi_{k}(x)<4\left(1-x^{2}\right)^{2} \sum_{k \geq 1} x^{2 k-2}=4(1+x)(1-x), \quad \forall x \in(0,1) .
$$

Let $x_{0}:=(\sqrt{113}-9) / 16<1$ and $h_{0}:=\log \left(1 / x_{0}\right)>0$. If $h \geq h_{0}$, then $x=\mathrm{e}^{h} \in\left(0, x_{0}\right]$ and $8 x^{2}+9 x-1 \leq 0$. In particular, we conclude that the bound (29) holds.

LEMma 23. If $h \geq h_{1}:=2 \log \frac{20}{9}$, then $E_{\omega}(h / 2) \neq 0$ for all $\omega \neq \pm \pi / 2$.

Proof. It is similar to the previous one. We introduce the positive quantities

$$
\tilde{\chi}_{k}=\tilde{\chi}_{k}(h):=\chi(k h+h / 2)=\frac{4 \cosh ^{2}(h / 2) \sinh ^{2}(h / 2)}{\cosh \left(\left(\frac{k}{2}+\frac{3}{4}\right) h\right) \cosh ^{2}\left(\left(\frac{k}{2}+\frac{1}{4}\right) h\right) \cosh \left(\left(\frac{k}{2}-\frac{1}{4}\right) h\right)} .
$$

Our goal is to prove that if $h \geq h_{1}$ and $\omega \neq \pm \pi / 2$, then

$$
\mathrm{e}^{\omega \mathrm{i}} E_{\omega}(h / 2)=2 \sum_{k \geq 0} \cos ((2 k+1) \omega) \tilde{\chi}_{k}=2\left(\tilde{\chi}_{0}+\sum_{k \geq 1} a_{k} \tilde{\chi}_{k}\right) \cos \omega \neq 0 .
$$

Here, we have used the symmetry $\tilde{\chi}_{-(k+1)}=\tilde{\chi}_{k}$ and we have introduced the notation

$$
a_{k}=a_{k}(\omega):=\frac{\cos (2 k+1) \omega}{\cos \omega} .
$$

That is, $a_{k}(\omega)=T_{2 k+1}(\cos \omega) / \cos \omega$, where $T_{n}(x)$ denotes the Chebyshev polynomial of first kind and degree $n$ defined by relation $T_{n}(\cos \omega)=\cos n \omega$. Now, using some standard properties of Chebyshev polynomials contained in [1, 22.5.29 \& 22.14.1], we realize that $\max _{\omega \in \mathbb{T}}\left|a_{k}(\omega)\right|=2 k+1$. Therefore, in order to prove the lemma it suffices to establish that

$$
\sum_{k \geq 1}(2 k+1) \tilde{\chi}_{k}(h)<\tilde{\chi}_{0}(h), \quad \forall h \geq h_{1}:=2 \log (20 / 9) .
$$

The rest of the proof is devoted to prove this bound. If we work with the multiplicative variable $x=\mathrm{e}^{-h / 2} \in(0,1)$, then $\tilde{\chi}_{0}=\tilde{\chi}_{0}(x)=4\left(1-x^{4}\right)^{2} / x\left(1+x^{3}\right)(1+x)^{3}$ and

$$
\tilde{\chi}_{k}=\tilde{\chi}_{k}(x)=\frac{4\left(1-x^{4}\right)^{2} x^{4 k-2}}{\left(1+x^{2 k+3}\right)\left(1+x^{2 k+1}\right)^{2}\left(1+x^{2 k-1}\right)}<4\left(1-x^{4}\right)^{2} x^{4 k-2}
$$

for any $k \geq 1$ and $x \in(0,1)$. In particular,

$$
\sum_{k \geq 1}(2 k+1) \tilde{\chi}_{k}(x)<4\left(1-x^{4}\right)^{2} \sum_{k \geq 1}(2 k+1) x^{4 k-2}=12 x^{2}\left(1-x^{4} / 3\right)
$$

for all $x \in(0,1)$. Hence, if there exists some $x_{1} \in(0,1)$ such that

$$
\frac{\left(1-x^{4}\right)^{2}}{1-x^{4} / 3}=: f(x) \geq g(x):=3 x^{3}\left(1+x^{3}\right)(1+x)^{3}, \quad \forall x \in\left(0, x_{1}\right]
$$




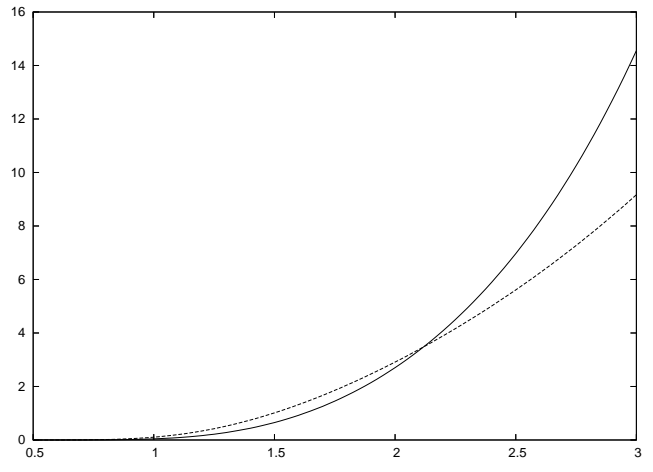

(a) Normal vertical scale

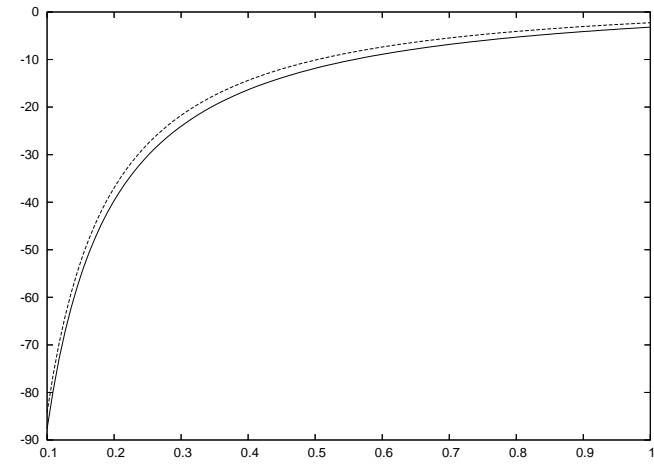

(b) Logarithmic vertical scale

FIG. 6. Graphs of the functions $m_{0}(h)=\min \left\{M_{0}(\omega, h): \omega \in \mathbb{T}\right\}$ (full curves) and $m_{1}(h)=$ $\min \left\{M_{1}(\omega, h): \omega \in \mathbb{T}\right\}$ (broken curves) in two different vertical scales.

then (30) holds for any $h \geq h_{1}:=2 \log \left(1 / x_{1}\right)$. The function $f(x)$ is increasing in the interval $(0,1)$, whereas $g(x)$ is decreasing in the same interval. On the other hand,

$$
f(9 / 20)=\frac{23543526721}{25250080000}>\frac{465593887647}{512000000000}=g(9 / 20) .
$$

Therefore, we can take $x_{1}:=9 / 20$.

\section{Appendix B. Numerical evidence on conjecture 15.}

In the proofs of the lemmas 22 and 23 , we have shown that the analytic functions $M_{j}: \mathbb{T} \times \mathbb{R}_{+} \rightarrow \mathbb{R}$ defined by

$$
M_{0}(\omega, h)=E_{\omega}(0), \quad M_{1}(\omega, h)=\frac{\mathrm{e}^{\omega \mathrm{i}} E_{\omega}(h / 2)}{2 \cos \omega}
$$

are positive when $h \geq h_{0}$ and $h \geq h_{1}$, respectively. We conjecture that, in fact, they are positive everywhere, which is equivalent to conjecture 15 .

We have displayed in figure 6 a strong numerical evidence about this conjecture. Concretely, we have numerically checked that the functions

$$
m_{j}: \mathbb{R}_{+} \rightarrow \mathbb{R}, \quad m_{j}(h)=\min \left\{M_{j}(\omega, h): \omega \in \mathbb{T}\right\}
$$

are positive in the range $1 / 10 \leq h \leq 3$. Greater values of $h$ are already covered by our analytical results. Smaller values of $h$ represent a computational challenge, because the functions $m_{j}(h)$ are exponentially small in $h$ as $h \rightarrow 0^{+}$, see subfigure $6(\mathrm{~b})$. This is a typical behaviour for splitting problems in weakly hyperbolic settings. The computation of such exponentially small splittings requires the use of a multiple precision arithmetic to mitigate the strong cancellations that take place in such problems. For instance, to compute the functions $m_{j}(h)$ at $h=1 / 10$ it is necessary to work with at least 50 digits.

\section{REFERENCES}

[1] M. Abramowitz and I. Stegun, Handbook of Mathematical Functions, Dover, New York, 1972.

[2] C. Amick, E. S. C. Ching , L. P. Kadanoff, And V. Rom-Kedar, Beyond all orders: singular perturbations in a mapping, J. Nonlinear Sci., 2 (1992), pp. 9-67. 
[3] V. I. Arnold And B. A. Khesin, Topological Methods in Hydrodynamics Applied Mathematical Sciences 125, Springer-Verlag, New York, 1998.

[4] R. B. Ash, Complex Variables, Academic Press, New York-London, 1971.

[5] S. Balasuriya, I. Mezić, And C. K. R. T. Jones, Weak finite-time Melnikov theory and 3D viscous perturbations of Euler flows, Physica D, 176 (2003), pp. 82-106.

[6] S. Balasuriya, Optimal perturbation for enhanced chaotic transport, Physica D, 202 (2005), pp. $155-176$.

[7] I. BAldomì And T. M. SEARA, Breakdown of heteroclinic orbits for some analytic unfoldings of the Hopf-zero singularity, J. Nonlinear Sci., 16 (2006), pp. 543-582.

[8] M. BAldomà And E. Fontich, Poincaré-Melnikov theory for $n$-dimensional diffeomorphisms, Appl. Math. (Warsaw), 25 (1998), pp. 129-152.

[9] H. W. Broer, C. Simó, And R. Vitolo, The Hopf-saddle-node bifurcation for fixed points of 3D-diffeomorphisms: analysis of a resonance 'bubble', preprint available in http://www.maia.ub.es/dsg/2007/.

[10] - The Hopf-saddle-node bifurcation for fixed points of 3D-diffeomorphisms: the Arnol'd resonance web, preprint available in http://www.maia.ub.es/dsg/2007/.

[11] H. W. Broer And G. Vegter, Subordinate Šil'nikov bifurcations near some singularities of vector fields having low codimension, Ergod. Theor. Dyn. Syst., 4 (1984), pp. 509-525.

[12] A. Delshams, Yu. Fedorov, and R. Ramírez-Ros, Homoclinic billiard orbits inside symmetrically perturbed ellipsoids, Nonlinearity, 14 (2001), pp. 1141-1195.

[13] A. Delshams and R. Ramírez-Ros, Poincaré-Melnikov-Arnold method for analytic planar maps, Nonlinearity, 9 (1996), pp. 1-26.

[14] - Melnikov potential for exact symplectic maps, Comm. Math. Phys., 190 (1997), pp. 213245.

[15] R. L. Devaney, Reversible diffeomorphisms and flows, Trans. Am. Math. Soc., 218 (1976), pp. 89-113.

[16] R. W. EAston, Computing the dependence on a parameter of a family of unstable manifolds: Generalized Melnikov formulas, Nonlinear Anal., 8 (1984), pp. 1-4.

[17] M. L. Glasser, V. G. Papageorgiou, and T. C. Bountis, Melnikov's function for twodimensional mappings, SIAM J. Appl. Math., 49 (1989), pp. 692-703.

[18] P. J. Holmes, Some remarks on chaotic particle paths in time-periodic, three-dimensional swirling flows, Contemp. Math., 28 (1984), pp. 393-404.

[19] Y. T. LaU And J. M. Finn, Dynamics of a three-dimensional incompressible flow with stagnation points, Physica D, 57 (1992), pp. 283-310.

[20] H. E. LomeLÍ, Saddle connections and heteroclinic orbits for standard maps, Nonlinearity, 9 (1996), pp. 649-668.

[21] - Applications of the Melnikov method to twist maps in higher dimensions using the variational approach, Ergodic Theory Dynam. Systems, 17 (1997), pp. 445-462.

[22] H. E. Lomelí AND J. D. MeISS, Heteroclinic primary intersections and codimension one Melnikov method for volume-preserving maps, Chaos, 10 (2000), pp. 109-121.

[23] - Heteroclinic intersections between invariant circles of volume-preserving maps, Nonlinearity, 16 (2003), pp. 1573-1595.

[24] H. E. Lomelí, J. D. Meiss, and R. Ramírez-Ros, Canonical Melnikov theory for diffeomorphisms, preprint arXiv:0706.2515.

[25] R. S. MACKAY, Transport in 3D volume-preserving flows, J. Nonlinear Sci., 4 (1994), pp. 329354.

[26] E. M. McMillan, A problem in the stability of periodic systems, in Topics in Modern Physics, E. Brittin and H. Odabasi, eds., Colorado Assoc. Univ. Press, Boulder, 1971, pp 219-244.

[27] I. Mezić And F. Sotiropoulos, Ergodic theory and experimental visualization of invariant sets in chaotically advected flows, Phys. Fluids, 14 (2002), pp. 2235-2243.

[28] I. MEZIĆ AND S. Wiggins, On the integrability and perturbation of three-dimensional fluid flows with symmetry, J. Nonlinear Sci., 4 (1994), pp. 157-194.

[29] P. Mullowney, K. Julien, And J. D. Meiss, Blinking rolls: chaotic advection in a threedimensional flow with an invariant, SIAM J. Appl. Dyn. Syst., 4 (2005), pp. 159-186.

[30] R. RAMíREZ-Ros, Exponentially small separatrix splittings and almost invisible homoclinic bifurcations in some billiard tables, Physica D, 210 (2005), pp. 149-179.

[31] T. Shinbrot, M. M. Alvarez, J. M. Zalc, and F. J. Muzzio, Attraction of minute particles to invariant regions of volume preserving flows by transients, Phys. Rev. Lett., 86 (2001), pp. $1207-1210$.

[32] F. Sotiropoulos, Y. Ventikos, and T. C. Lackey, Chaotic advection in three-dimensional stationary vortex-breakdown bubbles: Šl'nikov's chaos and the devil's staircase, J. Fluid Mech., 444 (2001), pp. 257-297. 
[33] E. T. Whittaker and G. N. Watson, A Course of Modern Analysis, Cambridge Univ. Press, Cambridge, 1927.

[34] S. L. ZigLIN, Splitting of separatrices, branching of solutions and nonexistence of an integral in the dynamics of a solid body, Trans. Moscow Math. Soc., 1 (1982), pp. 283-298. 